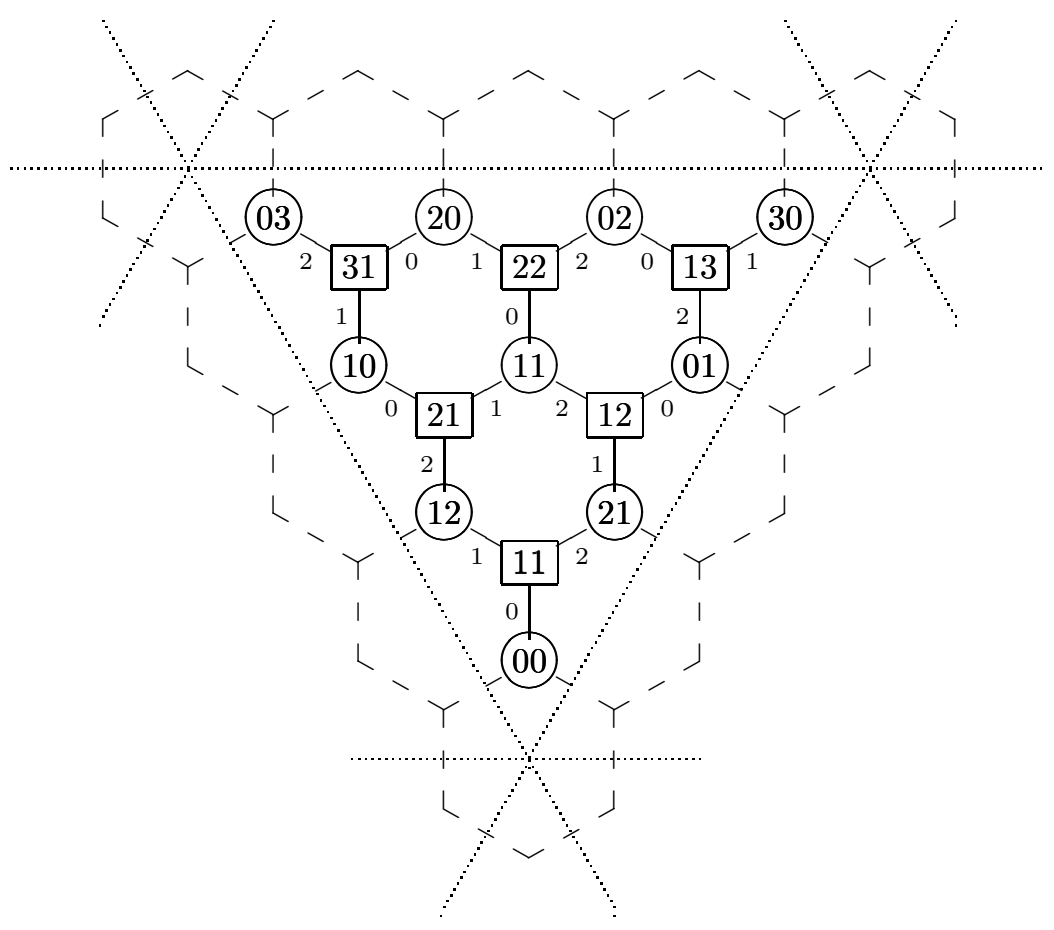

fig. 1

The $p^{\prime} / p=3 / 4$ admissible alcove.

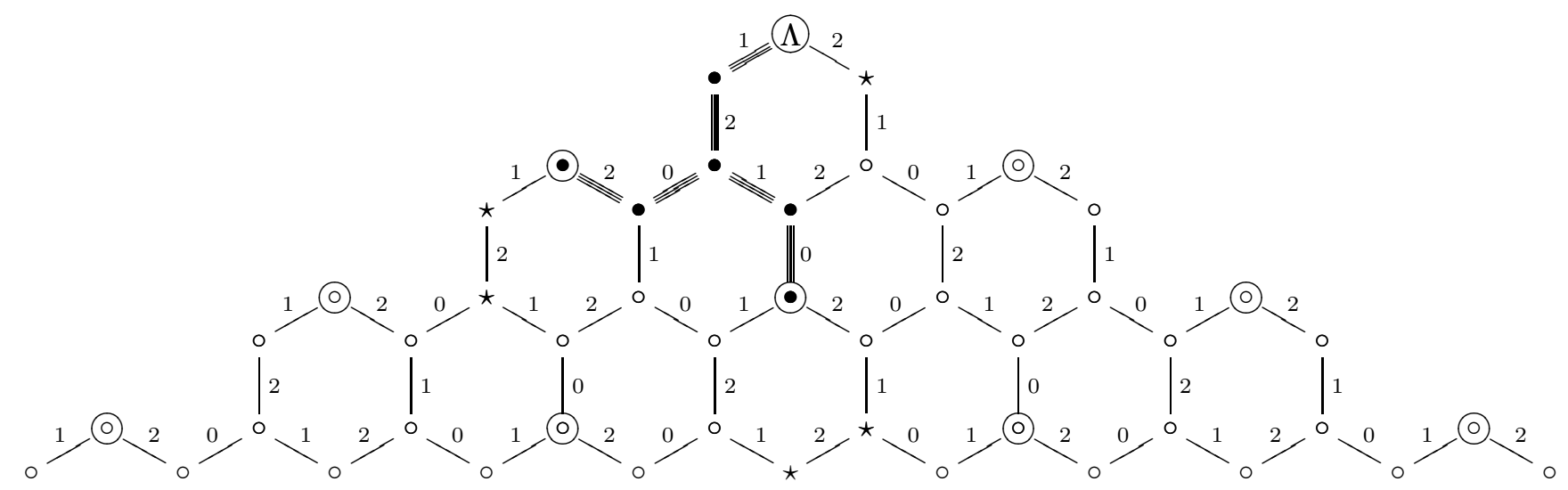

fig. 2

The begining of $\mathcal{W}_{\Lambda}^{+}$with the weight diagram $\mathcal{G}_{\Lambda}, \Lambda=[1,0]$. 


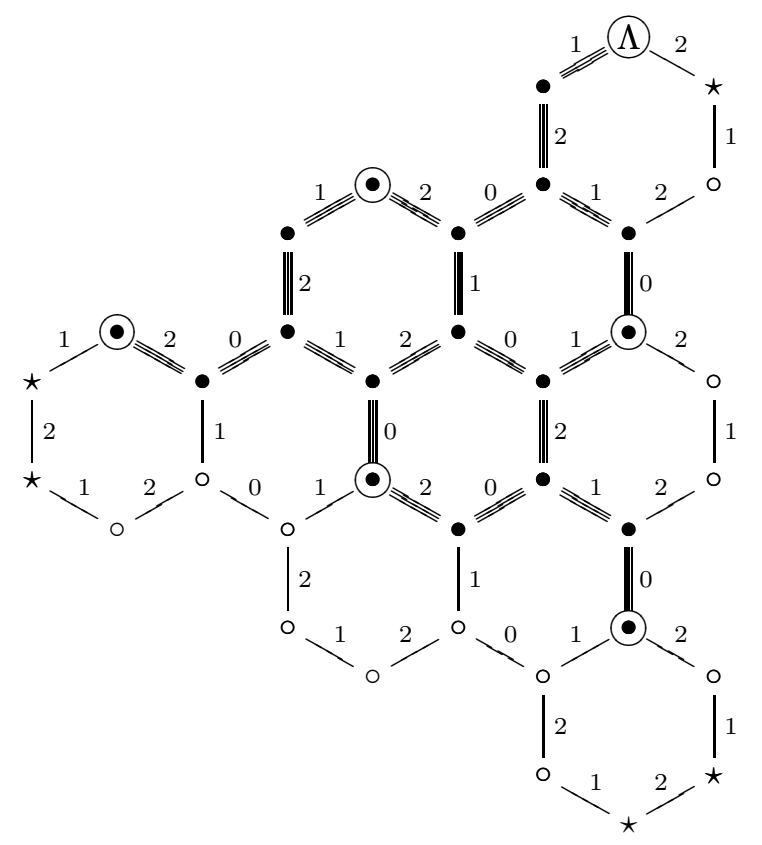

fig. 3

The weight diagram $\mathcal{G}_{[2,0]}$. The big circles indicate the $s l(3)$ counterpart diagram $-\kappa \Gamma_{(2,0)}$.

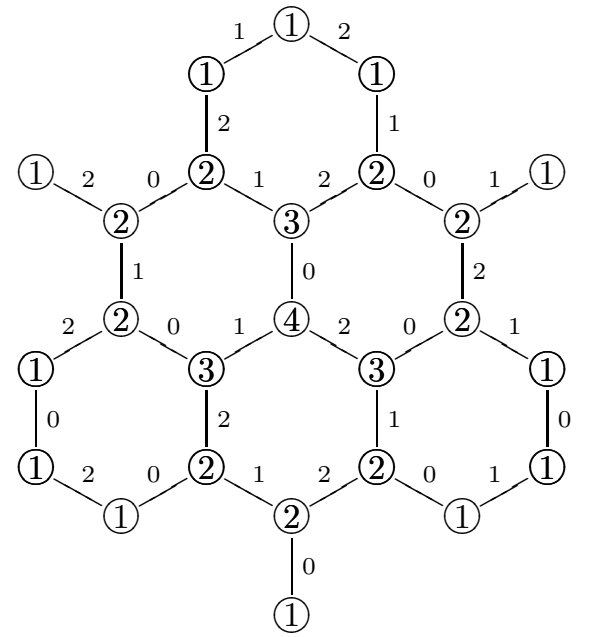

fig. 4

The $[1,1]$ diagram with weight multiplicities indicated in the circles. 


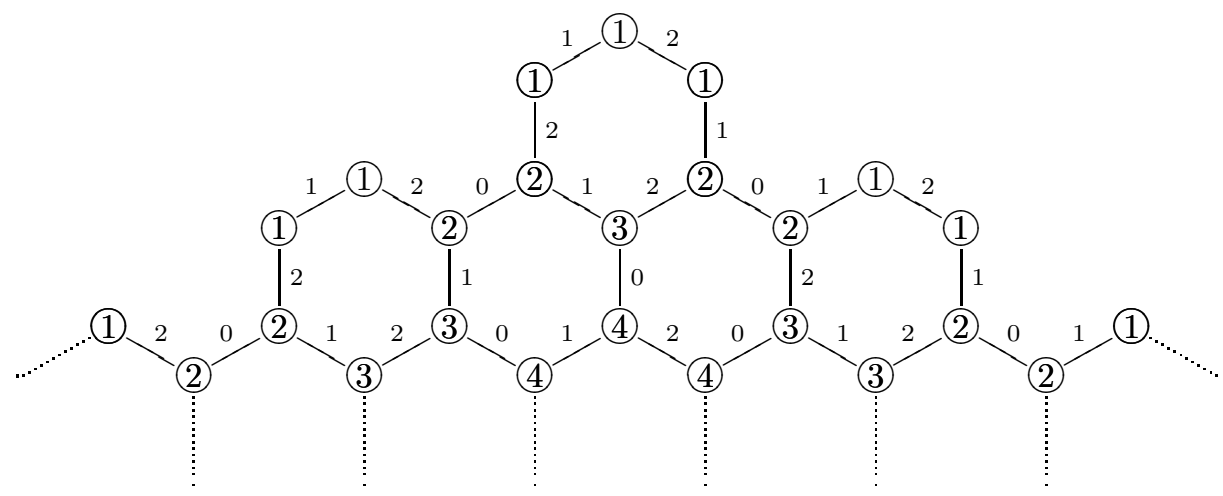

fig. 5

The begining of $\mathcal{W}^{+}$with multiplicities indicated in circles.

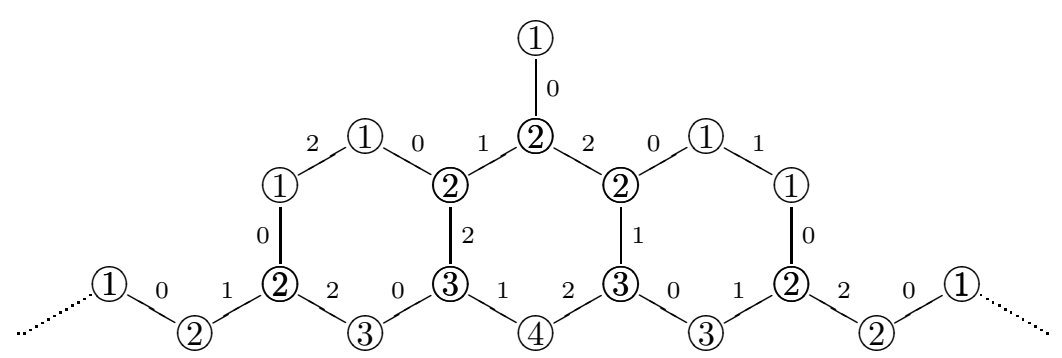

fig. 6

The beginning of $\mathcal{W}^{-}$with multiplicities in circles.

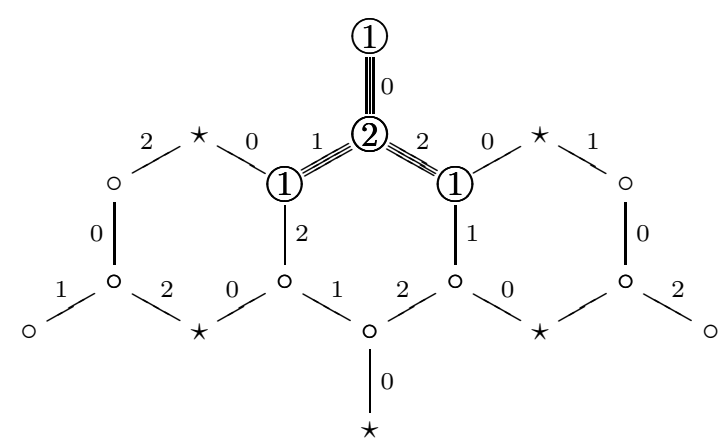

fig. 7

The beginning of $\mathcal{W}^{-}$with the resolution of $[[1,1]]$. The multiplicities of $\mathcal{G}_{[[1,1]]}$ are in circles. 


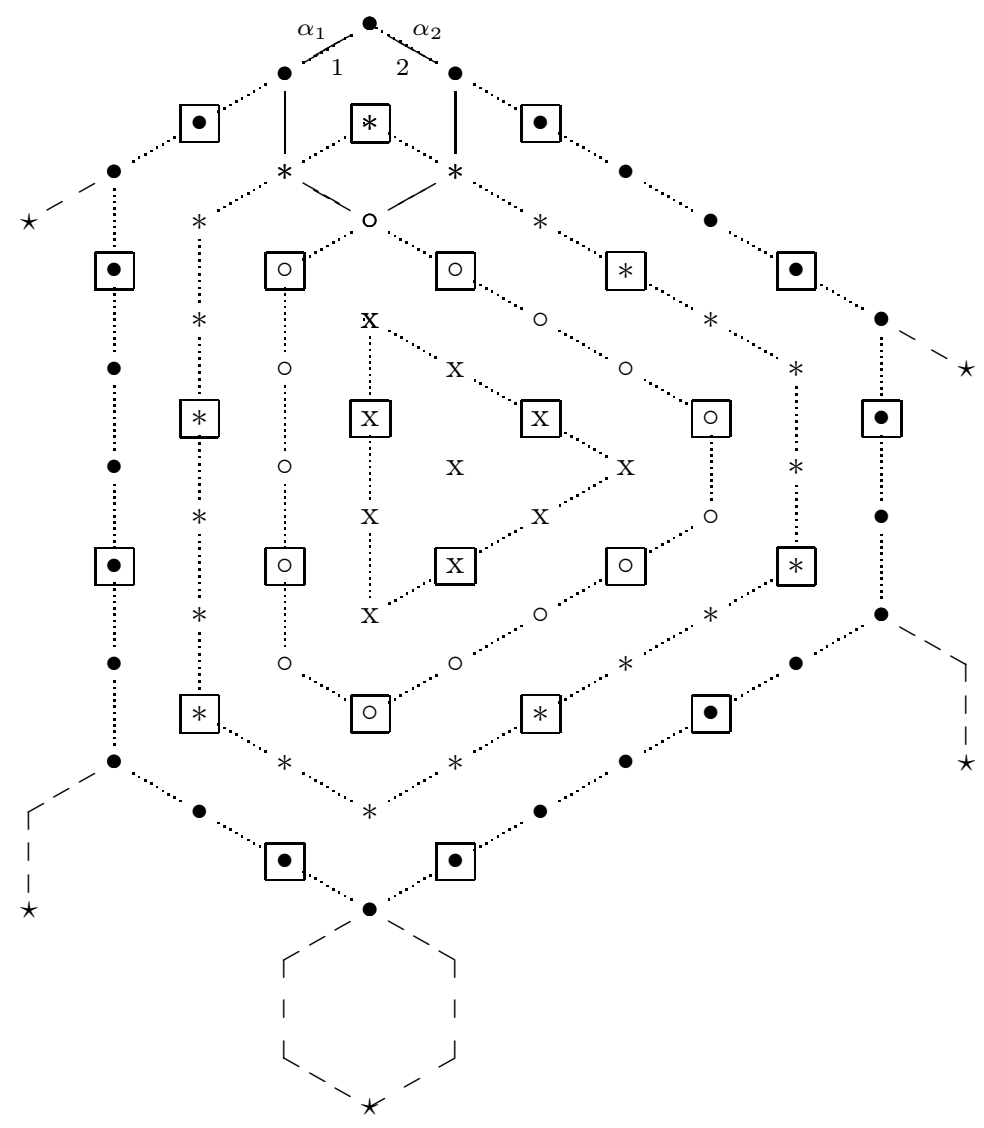

fig. 8

The weight diagram $\mathcal{G}_{-\kappa \lambda}$ for $\lambda=(1,2)$ with multiplicities $1,2,3,4$ depicted by $\bullet, *, \circ$, x, respectively; the edges of the elementary hexagons (except the first one) are omitted. The same picture describes the mapping of $\mathcal{G}_{-\kappa \lambda}$ into the $s l(3)$ weight diagram $\Gamma_{3 \lambda}$ - the points removed from the latter are indicated by squares. The stars $\star$ denote positions of singular vectors in the corresponding Verma modules. 


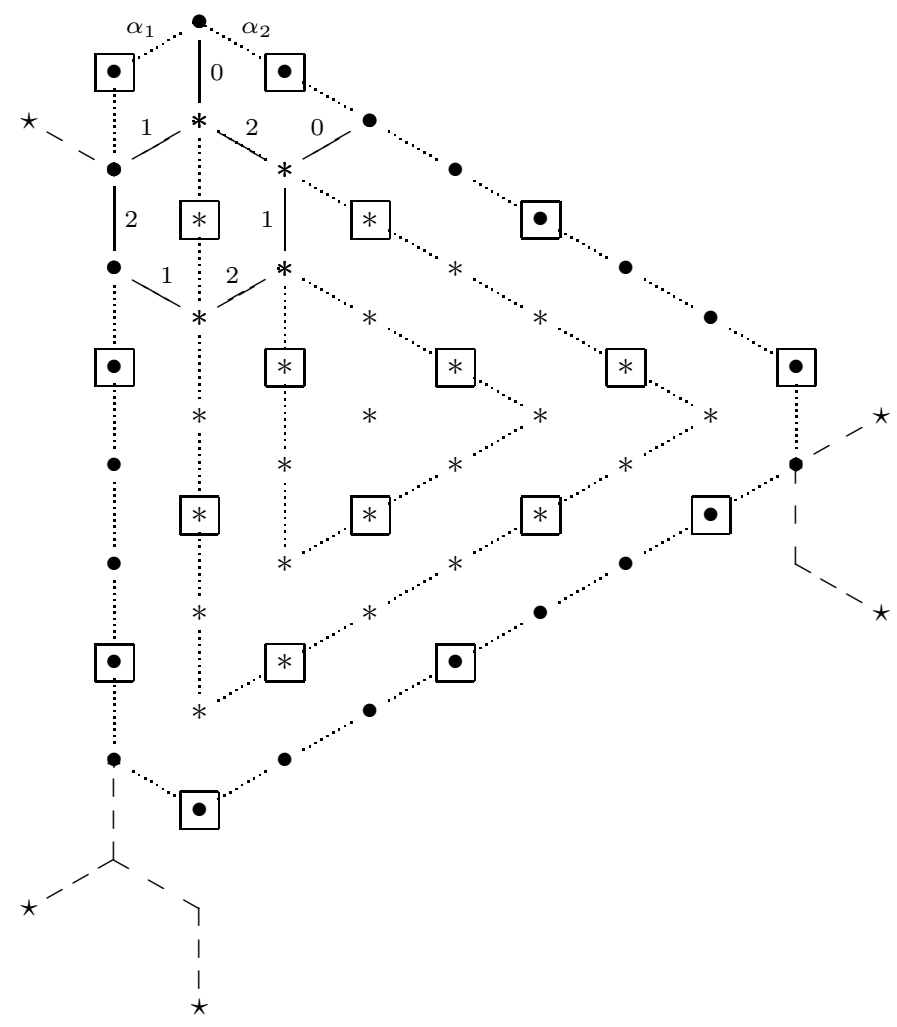

fig. 9

The weight diagram $\mathcal{G}_{w_{3} \cdot(-\kappa \lambda)}$ and its mapping into the $s l(3)$ weight diagram $\Gamma_{3 \lambda-2 \rho}$ for $\lambda=(1,3)$. The multiplicities 1,2 are depicted by $\bullet, *$, respectively.

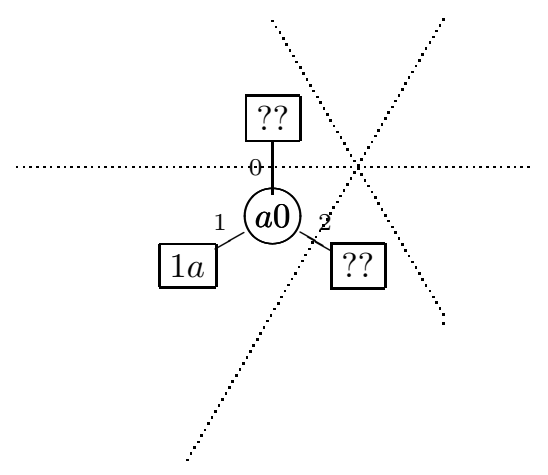

fig. 10

The fusion $[[1,1]] \otimes[a, 0]=[[1, a]]$, (for short $a=p-1$ ). The weights of the shifted diagram $\mathcal{G}_{[[1,1]]} \circ[a, 0]$ outside the admissible alcove are indicated by ??. Each one of them is of multiplicity one and together cancel the weight $[a, 0]$. 

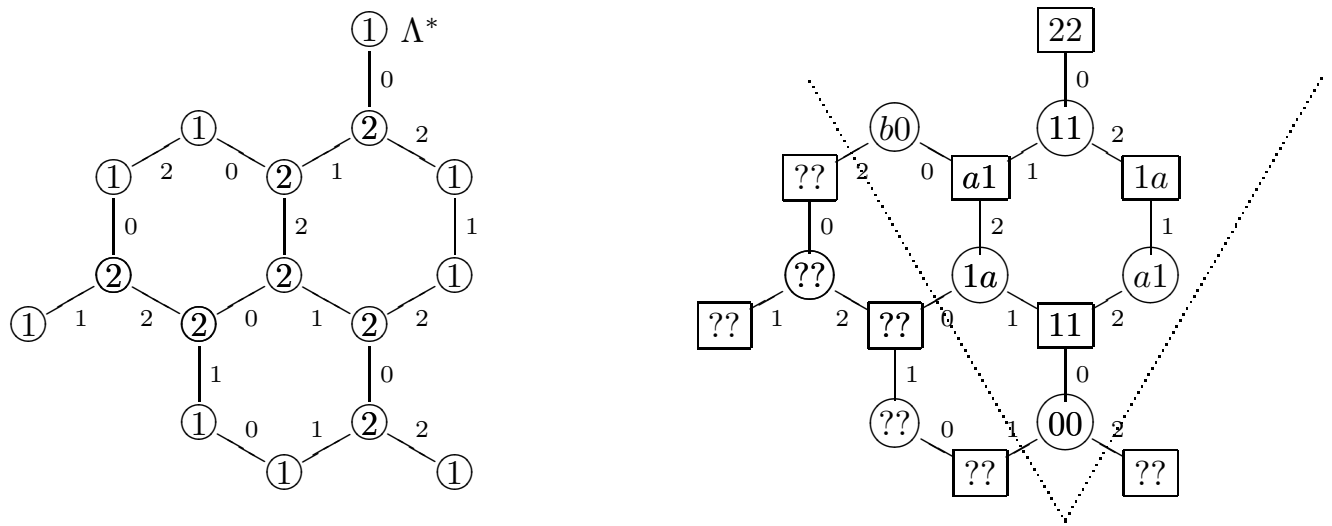

fig. 11

The fusion $[[2,1]] \otimes[0,1]=[[2,2]] \oplus[1,1] \oplus[[1, a]] \oplus[[1,1]] \oplus[a, 1], \quad a=p-2$.

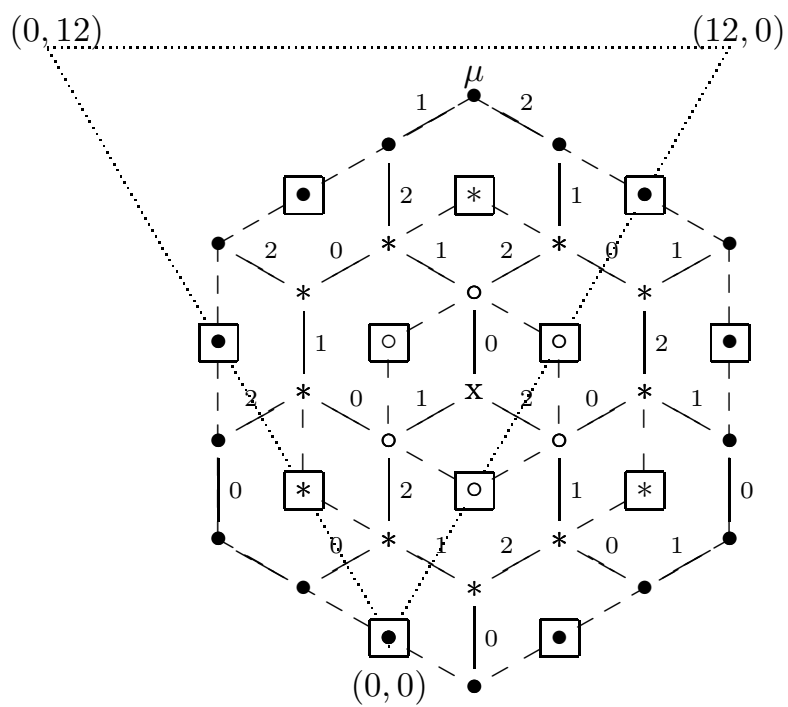

fig. 12

The comparison of the fusion $[1,1] \otimes[1,0]$ and $(3,3) \otimes(3,0)$. The convention for multiplicities is as in fig. 8 . 
hep-th/9709103

\title{
Fusion rules for admissible representations of affine algebras: the case of $A_{2}^{(1)}$
}

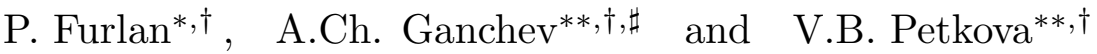 \\ Dipartimento di Fisica Teorica dell'Università di Trieste, Italy*, \\ Istituto Nazionale di Fisica Nucleare (INFN), Sezione di Trieste, Italy ${ }^{\dagger}$, \\ Institute for Nuclear Research and Nuclear Energy, Sofia, Bulgaria** \\ FB Physik, Uni Kaiserslautern, Germany $y^{\sharp}$
}

\begin{abstract}
We derive the fusion rules for a basic series of admissible representations of $\widehat{s l}(3)$ at fractional level $3 / p-3$. The formulae admit an interpretation in terms of the affine Weyl group introduced by Kac and Wakimoto. It replaces the ordinary affine Weyl group in the analogous formula for the fusion rules multiplicities of integrable representations. Elements of the representation theory of a hidden finite dimensional graded algebra behind the admissible representations are briefly discussed.
\end{abstract}

Pacs: 11.25.Hf; Keywords: fusion rules, admissible representations.

\section{Introduction}

The fusion rules (FR) are basic ingredient in any 2-dimensional conformal field theory [1], [2]. In [3] Awata and Yamada derived the FR for admissible irreducible representations of $\widehat{s l}(2)$ characterised by rational values of the level and the highest weights. The aim of this work is to extend this result to higher rank cases - for simplicity we present here the case $\widehat{s l}(3)$. More details and the general case $\widehat{s l}(n)$ will appear elsewhere. 
There is a formula for the FR multiplicities [4], [5], [6] in the case of integrable representations of $\widehat{s l}(n)$, equivalent to the better known Verlinde formula. It generalises the classical expression for the multiplicity of an irreducible representation in the tensor product of two finite dimensional $s l(n)$ representations, resulting from the Weyl character formula. The derivation in [6] was based essentially on the fundamental role played by the representation theory of $s l(n)$ and of its quantum counterpart $U_{q}(s l(n))$ at roots of unity. What complicates the problem under consideration is precisely the lack of knowledge of what is the finite dimensional algebra and its quantum counterpart, whose representation theory lies behind the fusion rules of admissible representations. In the case of $\widehat{s l}(2)$ Feigin and Malikov [7] have noticed that the relevant algebra is the superalgebra $\operatorname{osp}(1 \mid 2)$ and its deformed counterpart. Inverting somewhat the argumentation in [6], we shall try to show that the understanding of the fusion rules for admissible representations leads naturally to a set of finite dimensional representations of some (graded) algebra with well defined ordinary tensor product.

\section{Admissible weights.}

We start with introducing some notation. The simple roots of $\widehat{s l}(3)$ are $\alpha_{i}, i=$ $0,1,2$. The affine Weyl group $W$ is generated by the three simple reflections $w_{i}=w_{\alpha_{i}}$. For short let $w_{i j \ldots}=w_{i} w_{j} \ldots$ and furthermore $w_{\hat{1}}=w_{020}=w_{202}, w_{\hat{2}}=w_{010}=w_{101}$, $w_{\hat{0}}=w_{121}=w_{212}$, corresponding to the real positive roots $\alpha_{\hat{\imath}}=\delta-\alpha_{i}, \delta=\alpha_{0}+\alpha_{3}=$ $\alpha_{0}+\alpha_{1}+\alpha_{2}$. The elements $w_{\hat{\imath} \imath}$ and $w_{\hat{\imath} \hat{\imath}}$ constitute (elementary) affine translations $t_{ \pm \bar{\alpha}_{i}}$, where $\bar{\alpha}_{0}=-\alpha_{3}, \bar{\alpha}_{i}=\alpha_{i}, i=1,2$. Denote the fundamental weights by $\omega_{0}, \omega_{i}+\omega_{0}$, $i=1,2, \omega_{i}$ being the horizontal $\operatorname{sl}(3)$ subalgebra fundamental weights. Let $P=\sum_{i=1}^{2} \mathbb{Z} \omega_{i}$, $P_{+}=\sum_{i=1}^{2} \mathbb{Z}_{+} \omega_{i}, P_{++}=\sum_{i=1}^{2} \mathbb{N} \omega_{i}$ be the $s l(3)$ weight lattice, the integral dominant and strictly integral dominant weights and $P_{+}^{k}=\left\{\lambda \in P_{+},\left\langle\lambda, \alpha_{3}\right\rangle \leq k\right\}, P_{++}^{k+3}=\{\lambda \in$ $\left.P_{++},\left\langle\lambda, \alpha_{3}\right\rangle \leq k+2\right\}\left(k \in \mathbb{Z}_{+}\right)$, the corresponding alcoves. For short we shall denote all $\widehat{s l}(3)$ weights at an arbitrary level $k$ with their horizontal projections $\Lambda=\sum_{i=1,2}\left\langle\Lambda, \alpha_{i}\right\rangle \omega_{i}$. Accordingly $w \cdot \Lambda$ will indicate the horizontal projection of the shifted (by $\omega_{1}+\omega_{2}+3 \omega_{0}$ ) action on $\Lambda+k \omega_{0}$ of the affine Weyl group. For nonnegative integer $k \lambda \in P_{+}^{k}\left(P_{++}^{k+3}\right)$ runs over highest weights (shifted by $\rho=\omega_{1}+\omega_{2}$ highest weights) of integrable representations at level $k$. 
Given a fractional level $k$ such that $\kappa \equiv k+3=p^{\prime} / p$ with $p, p^{\prime}$ coprime integers and $p^{\prime} \geq 3$, the set of admissible weights of $\widehat{s l}(3)$ is defined [8] as

$$
P_{p^{\prime}, p}=\left\{\lambda^{\prime}-\lambda \kappa \mid \lambda^{\prime} \in P_{+}^{p^{\prime}-3}, \lambda \in P_{+}^{p-1}\right\} \cup\left\{w_{3} \cdot\left(\lambda^{\prime}-\lambda \kappa\right) \mid \lambda^{\prime} \in P_{+}^{p^{\prime}-3}, \lambda \in P_{++}^{p+1}\right\} .
$$

Due to invariance with respect to a Coxeter element generated subgroup of the horizontal Weyl group $\bar{W}$ (see [8] for details) the domain in (2.1) can be equivalently represented using other elements $w \in \bar{W}$.

We shall refer to $P_{p^{\prime}, p}$ as the admissible alcove and to its first, second subsets as its first, second leaf. We have reversed the traditional notation putting the prime on the integer part of the weights and leaving the fractional part unprimed, the reason being that in this paper we shall restrict ourselves mostly to the particular series of admissible representations defined by $p^{\prime}=3, p \geq 4$, in which only $\lambda^{\prime}=0$ survives in the pairs $\left(\lambda^{\prime}, \lambda\right)$ of $s l(3)$ weights appearing in (2.1). The addmissible alcove $P_{3, p}$ (to be called sometimes "the double alcove") is described by a collection of $\left(\begin{array}{c}p+1 \\ 2\end{array}\right)+\left(\begin{array}{c}p \\ 2\end{array}\right)=p^{2}$ integrable weights at integer levels $p-1$ and $p-2$, entering the fractional parts of the weights of the first and second leaf respectively. The choice $p^{\prime}=3$ is not very restrictive since the novel features of the fusion rules are essentially governed by the fractional part of the admissible highest weights and furthermore the subseries $p^{\prime}=3$ is interesting by itself. Shorthand notation $\left[n_{1}, n_{2}\right]$ and $\left[\left[n_{1}, n_{2}\right]\right], n_{i}=\left\langle\lambda, \alpha_{i}\right\rangle$, will be used for the weights $\Lambda=-\lambda \kappa$ on the $1^{\text {st }}$ leaf and $\Lambda=w_{3} \cdot(-\lambda \kappa)$ on the $2^{\text {nd }}$ leaf of $P_{3, p}$ in (2.1), respectively; $n_{3}=n_{1}+n_{2}$. For $\Lambda \in P_{3, p}$ we shall exploit the automorphism groups $\mathbb{Z}_{3}$ of the alcoves $P_{+}^{p-1}$ and $P_{++}^{p+1}$ generated by

$$
\begin{aligned}
& \sigma: \Lambda=\left[n_{1}, n_{2}\right] \mapsto \sigma(\Lambda)=\left[p-1-n_{3}, n_{1}\right], \\
& \sigma: \Lambda=\left[\left[n_{1}, n_{2}\right]\right] \mapsto \sigma(\Lambda)=\left[\left[n_{2}, p+1-n_{3}\right]\right] .
\end{aligned}
$$

The $\widehat{s l}(3)$ Verma modules labelled by admissible highest weights are reducible, with submodules determined by the Kac-Kazhdan theorem [9]. In general for an arbitrary weight $\Lambda$ let $M_{i}=\left\langle\Lambda+\rho+\kappa \omega_{0}, \alpha_{i}\right\rangle, i=0,1,2$. If for a fixed $i=0,1,2$, the projection $M_{i}$ can be written as $M_{i}=m_{i}^{\prime}-\left(m_{i}-1\right) \kappa$ (or as $\left.M_{i}=-m_{i}^{\prime}+m_{i} \kappa\right)$, where $m_{i}^{\prime}, m_{i} \in \mathbb{N}$, there is a singular vector of weight $w_{\beta_{i}} \cdot \Lambda$ (or $w_{\beta_{\hat{\imath}}} \cdot \Lambda$ ) in the Verma module of highest weight $\Lambda$. It corresponds to the affine real positive root $\beta_{i}=\left(m_{i}-1\right) \delta+\alpha_{i}\left(\right.$ or $\left.\beta_{\hat{\imath}}=m_{i} \delta-\alpha_{i}\right)$ respectively. These Weyl reflections can be represented as

$$
w_{\beta_{i}}=t_{-\left(m_{i}-1\right) \bar{\alpha}_{i}} w_{i}=w_{i}\left(w_{\hat{\imath} i}\right)^{m_{i}-1}, \quad w_{\beta_{\hat{\imath}}}=t_{\left(m_{i}-1\right) \bar{\alpha}_{i}} w_{\hat{\imath}}=w_{\hat{\imath}}\left(w_{i \hat{\imath}}\right)^{m_{i}-1}, \quad i=0,1,2 .
$$

1 We neglect the terms $d \delta$ in the full admissible highest weights as irrelevant to our purposes. 
The corresponding singular vectors were constructed in [10. To a decomposition of the reflections (2.3) into a product of simple reflections corresponds a monomial of the lowering generators of $\widehat{s l}(3)$, namely every $w_{i}, i=0,1,2$, is substituted by $E^{-\alpha_{i}}$ to an appropriate (in general complex) power, see [11] for more explicit presentation in the case of $\widehat{s l}(3)$.

Consider the Weyl groups $\bar{W}^{\lambda}$ and $W^{\lambda}$ generated for the representations on the first (second) leaf of (2.1) by the reflections $w_{\beta_{i}}\left(w_{\beta_{\hat{\imath}}}\right), i=1,2, \beta_{i}=\left\langle\lambda, \alpha_{i}\right\rangle \delta+\alpha_{i} \quad\left(\beta_{\hat{\imath}}=\right.$ $\left.-\left\langle\lambda, w_{3}\left(\alpha_{i}\right)\right\rangle \delta-\alpha_{i}\right)$ and $w_{\beta_{i}}\left(w_{\beta_{\hat{\imath}}}\right), i=0,1,2$, with $\beta_{0}=\left(p-1-\left\langle\lambda, \alpha_{3}\right\rangle\right) \delta+\alpha_{0}\left(\beta_{\hat{0}}=\right.$ $\left.\left(p+1-\left\langle\lambda, \alpha_{3}\right\rangle\right) \delta-\alpha_{0}\right)$ respectively. Similarly one defines 4 more variants corresponding to the various equivalent ways of representing the admissible alcove. The groups $\bar{W}^{\lambda}$ and $W^{\lambda}$ are isomorphic to $\bar{W}$ and $W$ and were introduced and exploited by Kac and Wakimoto (KW) [8] in the study of the characters of admissible representations. We shall refer to these groups, which will play a crucial role in what follows, as the KW groups. Apparently any element of the KW group $W^{\lambda}$ depends on (and is determined by) the point on which it acts, so in a sense this is a "local" group acting on the double alcove and "spreading" it much in the same way as the ordinary affine Weyl group acts on the fundamental integrable alcove. This is more transparent using an alternative description of $P_{3, p}$.

\section{Alternative description of the admissible alcove $P_{3, p}$. Affine Weyl group graph replacing the weight lattice.}

First recall that the affine Weyl group $W$ can be represented as a graph to be denoted $\mathcal{W}$. This is the well known "honeycomb" lattice (which we saw for the first time in [12) covering the plane, consisting of hexagons with links labelled by the three elementary reflections $w_{i}, i=0,1,2$, - so that at each vertex meet 3 (always different) links, while along any hexagon two of the three reflections appear at an alternating order. (All figures in this paper depict some finite part of this graph; the labels $0,1,2$ on the edges correspond to the reflections $w_{0}, w_{1}, w_{2}$.) The three different hexagons appearing on the graph depict the three 6 -term relations among the generators $w_{i}, i=0,1,2$, e.g., $w_{121}=w_{212}$. Thus if we choose an origin, a vertex of this graph, to represent the identity element of $W$, then the vertices are in bijective correspondence with the elements of $W$, while the different paths connecting a vertex with the origin depict the different presentations of an element of $W$ in terms of the generators $w_{i}$.

The dual lattice has as vertices the centers of the hexagons, 6 dual links meet at a dual vertex, and the elementary cells are triangles centered at the vertices of the hexagonal 
lattice. For given $\kappa=3 / p$ consider a triangle in the dual lattice the sides of which consist of $p$ dual links. The weights in $P_{3, p}$ can be arranged on the piece of $\mathcal{W}$ enclosed by this triangle. This "big" alcove consists of $p^{2}$ elementary triangles, equivalently $p^{2}$ vertices of the honeycomb. The three vertices sitting at the three corners are connected with the other points of the alcove by the three possible links $0,1,2$. Choose the corner vertex that is connected by a 0 -link as the origin and assign to it the weight $[0,0]$. To the other vertices of the alcove assign weights by the shifted action of $W$. The vertices at an even number of links from the origin accommodate weights on the $1^{\text {st }}$ leaf while the ones at an odd number - on the $2^{\text {nd }}$ leaf. The example $p=4$ is depicted on fig. 1 . The three lines of the dual lattice cutting out the alcove are indicated by dotted lines. The weights $[n, m]$ are in circles, the weights $[[n, m]]$ in boxes. (In general the first few points are e.g., $w_{20} \cdot[0,0]=[p-2,1], w_{120} \cdot[0,0]=[[1, p-2]], w_{0120} \cdot[0,0]=[0, p-3]$, etc., obtained by adding and subtracting $p^{\prime}=p \kappa$.) The border lines (paths) of the big alcove consist of highest weights $\Lambda$ such that $w_{i} \cdot \Lambda \notin P_{3, p}$, or $w_{\hat{\imath}} \cdot \Lambda \notin P_{3, p}$, for some $i=0,1,2$ - they precisely exhaust the weights labelling the border points of the two alcoves $P_{+}^{p-1}, P_{++}^{p+1}$.

"Reflecting" $\Lambda$ in the three boundary lines enclosing the alcove we land at a weight of a singular vector in the $\widehat{s l}(3)$ Verma module of highest weight $\Lambda$. These reflections generate a group that coincides with $W^{\lambda}$. The alcove is a fundamental domain with respect to the action of this group. In this realisation the affine Weyl group graph $\mathcal{W}$ plays the role of "weight lattice". The choice of the reflecting "hyperplanes" described by $\left\langle\Lambda^{\prime}+\rho+\kappa \omega_{0}, \beta_{i}\right\rangle=0$ and $\left\langle\Lambda^{\prime}+\rho+\kappa \omega_{0}, \beta_{\hat{\imath}}\right\rangle=0, i=0,1,2$ (i.e., their precise identification with the three planes cutting the big triangle) depends on the value of $p$ and the given highest weight $\Lambda$ in the alcove.

As it is clear from fig. 1 . any "big" alcove $P_{3, p}$ can be canonically mapped into $P_{++}^{3 p}$, the admissible highest weights being identified with a subset of the triality 0 integrable (shifted by $\rho$ ) highest weights at level $3 p-3$.

\section{Decoupling of states generated by singular vectors. Affine Weyl group graph replacing the root lattice.}

In view of the complexity of the Malikov-Feigin-Fuks (MFF) singular vectors in the case $\widehat{s l}(n), n>2$, it is not realistic to repeat the derivation for $n=2$ in [3], by solving all equations for the 3-point blocks expressing the decoupling of the submodules generated by 
singular vectors in the admissible Verma modules. Instead we shall reduce the problem to the solution of some basic subset of equations.

Our first step is the solution of the simplest equations arising from the singular vectors in the Verma module labelled by the "fractional fundamental" weight $f:=[1,0]=-\kappa \omega_{1}$ (for short we will call it fundamental). Using the standard realisation of the induced representations of $S L(3)$ in terms of functions $\varphi(x, y, z)$ depending on ("isospin") coordinates $(x, y, z)$, described by a triangular matrix in the Gauss decomposition of the elements of $S L(3)$, we realise the generators by the corresponding differential operators (see, e.g., [13]). The isospin coordinates (as well as the space-time coordinates) of the first and third fields in the 3 -point functions are fixed to 0 and " $\infty$ ". For the weight $f$ (labelling the field at the first point) there are two singular vectors corresponding to the roots $\beta_{1}=\delta+\alpha_{1}$ and $\beta_{2}=\alpha_{2}$ and determined by $(2.3)$ with $w_{\beta_{1}}=w_{12021}=w_{1 \hat{1} 1}, w_{\beta_{2}}=w_{2}$. Since the second of the singular vectors reduces simply to the $s l(3)$ generator $E^{-\alpha_{2}}=-\left(\partial_{y}+x \partial_{z}\right)$ of $y$ translations, which annihilates the monomials of type $x^{a}(x y-z)^{c}$, we look for a solution of the equation corresponding to the first MFF operator (see [11] for details) in terms of such monomials (in general $-x^{a} y^{b}(x y-z)^{c} z^{d}$ ) - it reduces to an algebraic system of two equations for the unknowns $(a, c)$. Due to Ward identities corresponding to the two Cartan generators the powers $a, b$ (or $(a, b, c, d)$ in general) are expressed in terms of the three weights $\Lambda^{(i)}, i=1,2,3$, labelling the fields in a 3 -point function

$$
\begin{gathered}
-2 a+b-c-d+\left\langle\Lambda^{(1)}+\Lambda^{(2)}, \alpha_{1}\right\rangle=\left\langle\Lambda^{(3)}, \alpha_{1}\right\rangle, \\
a-2 b-c-d+\left\langle\Lambda^{(1)}+\Lambda^{(2)}, \alpha_{2}\right\rangle=\left\langle\Lambda^{(3)}, \alpha_{2}\right\rangle .
\end{gathered}
$$

Taking $\Lambda^{(1)}=f$ and $\Lambda^{(2)}=\Lambda^{\prime},\left\langle\Lambda^{(2)}+\rho, \alpha_{i}\right\rangle=M_{i}, i=1,2$, we find 7 solutions for the resulting representation $\Lambda^{(3)}$ in the fusion $f \otimes \Lambda^{\prime}$, described by the values $(a, c)=(0,0)$, $\left(M_{1}-\kappa, 0\right),\left(-M_{2}, M_{3}-\kappa\right),\left(0, M_{3}-\kappa\right),\left(-M_{2}, M_{2}-\kappa\right),(-\kappa, 0),(0,-\kappa)$, while the corresponding highest weights read:

\section{Proposition 1:}

$$
\begin{gathered}
f \otimes \Lambda^{\prime}=\left(f+\Lambda^{\prime}\right) \oplus w_{1} \cdot\left(f+\Lambda^{\prime}\right) \oplus w_{21} \cdot\left(f+\Lambda^{\prime}\right) \oplus w_{121} \cdot\left(f+\Lambda^{\prime}\right) \\
\oplus w_{021} \cdot\left(f+\Lambda^{\prime}\right) \oplus w_{2021} \cdot\left(f+\Lambda^{\prime}\right) \oplus w_{0121} \cdot\left(f+\Lambda^{\prime}\right)
\end{gathered}
$$

The result (4.2) holds for arbitrary generic admissible highest weight $\Lambda^{\prime} \in P_{p^{\prime}, p}$ (i.e., such that all representations in the r.h.s of (4.2) belong to the admisible domain (2.1) ) and arbitrary $p^{\prime}$ and $p \geq 4$, i.e., we do not restrict here to the subseries $p^{\prime}=3$. Analogously 
the fusion rule of the conjugate representation $f^{*}=[0,1]$ with a generic $\Lambda^{\prime}$ reads as in (4.2) with $w_{1}$ and $w_{2}$ interchanged.

Taking $\Lambda^{\prime}=0$, the set of 7 weights in the r.h.s. of (4.2) replaces the three weights of the $s l(3)$ fundamental representation. The latter have clear counterparts in (4.2) represented by the highest weight $f=-\kappa \omega_{1}$ and the two last weights $w_{\hat{1} 1} \cdot f=-\kappa\left(\omega_{1}-\alpha_{1}\right), w_{0 \hat{0}} \cdot f=$ $-\kappa\left(\omega_{1}-\alpha_{3}\right)$.

We can visualize this 7 point "weight diagram", to be denoted $\mathcal{G}_{f}$ (or its "shifted" by $\Lambda^{\prime}$ version defined by the weights in the r.h.s. of (4.2), to be denoted $\mathcal{G}_{f} \circ \Lambda^{\prime}$ ) as a collection of points on the affine Weyl group graph $\mathcal{W}$, see fig. 2. Identifying a reference point on this graph with a highest weight $\Lambda=-\kappa \lambda$ on the first leaf of $P_{p^{\prime}, p}$ (in our case $\Lambda=f$ ) we build its "positive" part $\mathcal{W}_{\Lambda}^{+}$in close analogy with the set $Q_{\lambda}^{+}=\lambda-Q^{+}$, $Q^{+}=\sum_{i=1,2} \mathbb{Z}_{+} \alpha_{i} \subset Q, Q$ being the root lattice of $\operatorname{sl}(3)$ (see also sect. 6 below). On fig. 2 we have depicted the beginning of $\mathcal{W}_{f}^{+}$. Weights from the sublattice $\Lambda-(-\kappa) Q^{+}$of $\mathcal{W}_{\Lambda}^{+}$ are denoted by big circles. They correspond to sequences of translations $\kappa \alpha_{j}, j=1,2,3$, of $\Lambda$ along the $\operatorname{sl}(3)$ positive roots, each elementary translation being represented by a 4-pieces path on $\mathcal{W}_{\Lambda}^{+}$. Two such paths (or one on the border lines) replace a link on $Q_{\lambda}^{+}$ joining two weights which differ by a root $-\alpha_{j}$. Now $\mathcal{G}_{f}$ is the finite part of $\mathcal{W}_{f}^{+}$consisting of all points "enclosed" by the three points on $-\kappa \Gamma_{(1,0)}$, where $\Gamma_{(1,0)}$ is the weight diagram of the $\operatorname{sl}(3)$ counterpart of $f$. The points of $\mathcal{G}_{f}$ on fig. 2 are connected by bold links.

On fig. 3 we have depicted the weight diagram $\mathcal{G}_{[2,0]}$. The same picture with the reference point $\Lambda$ substituted by $\Lambda+\Lambda^{\prime}$ represents the shifted weight diagram $\mathcal{G}_{[2,0]} \circ \Lambda^{\prime}$ obtained solving the decoupling equations for the fusion of $[2,0]$ with a generic $\Lambda^{\prime} \in P_{p^{\prime}, p}$.

The working hypothesis which emerges after analysing the decoupling equations for a couple of examples is that in general these generalised weight diagrams $\mathcal{G}_{\Lambda}$ for highest weight $\Lambda=-\kappa \lambda$ from the $1^{\text {st }}$ leaf of the admissible alcove are obtained according to the following rule. Let $\Gamma_{\lambda} \subset Q_{\lambda}^{+}$be the weight diagram of the $\operatorname{sl}(3)$ irreducible representation of highest weight $\lambda$. Embed $-\kappa \Gamma_{\lambda}$ (as a set of points) into the sublattice $\Lambda+\kappa Q^{+}$of $\mathcal{W}_{\Lambda}^{+}$. Draw all paths on $\mathcal{W}_{\Lambda}^{+}$, starting from the highest weight $\Lambda$, that lie within the borders of $-\kappa \Gamma_{\lambda}$, including the "border" path connecting all points on the outmost (multiplicity one) layer of $-\kappa \Gamma_{\lambda}$. The diagram $\mathcal{G}_{\Lambda}$ is the resulting finite set of weights on $\mathcal{W}_{\Lambda}^{+}$.

2 The solutions of the equations corresponding to the singular vectors expressed by $E^{-\alpha_{2}},\left(E^{-\alpha_{1}}\right)^{2}$ in the $\operatorname{sl}(3)$ case are accordingly given by the same monomials with $(a, c)=(0,0),(1,0),(0,1)$. For a general computation of this kind, recovering the $s l(3)$ representation spaces, see 13. 
Besides the series $[0, n]$, or $[n, 0]$, little can be said at this point about the precise values of the multiplicities assigned to the points of the general weight diagrams. The ansatz with monomials (images of 3 -point functions at $0,(x, y, z)$ and " $\infty$ ") used in the analysis of the singular vectors equations does not reproduce all expected multiplicities and furthermore gives controversial results using different "shifting" representations. 3 The first example with nontrivial multiplicities is provided by $\Lambda=[1,1]$ (the analog of the $s l(3)$ adjoint representation $(1,1))$. Its weight diagram is depicted on fig. 4 . To find the indicated weight multiplicities we have followed a different strategy which recovers as well the general FR multiplicities.

\section{Fundamental fusion graphs. Pasquier-Verlinde type formula for the FR multiplicities.}

The rule (4.2) describes the fusion of the fundamental representation with a generic representation $\Lambda^{\prime}$ - in general one expects truncations, i.e., some of the resulting representations in the fusion lie outside the admissible alcove and furthermore some admissible representations are forbidden. To determine them one has to analyse the additional equations resulting from the decoupling of singular vectors in the Verma module of highest weight $\Lambda^{\prime}$, taking now the fundamental one as the "shifting" representation. This implies that we select and check only the solutions of these equations corresponding to weights belonging to the 7 point "shifted" fundamental diagram $\mathcal{G}_{f} \circ \Lambda^{\prime}$; they are described by some sets of the powers $(a, b, c, d)$ (one of them always equal to zero), subject of the relations (4.1).

Before starting this analysis we first find that the representations described by the $\mathbb{Z}_{3}$ orbit of the identity element $\mathbf{1}=[0,0]$, i.e., the corner points of the big alcove, are simple currents, i.e., their fusion with an arbitrary representation on the first or second leaf of the double alcove produces only one representation living on the same leaf, namely the nontrivial FR multiplicities $N_{a \sigma(\mathbf{1})}^{b}$ read

$$
N_{a \sigma(\mathbf{1})}^{b}=\delta_{b \sigma(a)} \Rightarrow N_{a \sigma(b)}^{\sigma(c)}=N_{a b}^{c}
$$

3 On the other hand an alternative approach based on the full invariant 3-point functions and singular vectors realised by "right action" (Verma module generating) $\widehat{s l}(3)$ generators is technically difficult to be implemented even in the simplest examples. 
where we have denoted by the same letter the action of the two (different) $\mathbb{Z}_{3}$ groups defined in (2.2). The second equality in (5.1) is a consequence of the first and the associativity of the fusion rules and enables one, as in the integrable case, to reduce the computation of the FR on any $\mathbb{Z}_{3}$ orbit to the FR of one representative of that orbit. The simple current property is established solving the relevant singular vector equations. E.g., for $\sigma(\mathbf{1})=[p-1,0]$ they correspond to the roots $\beta_{\hat{1}}, \beta_{0}, \beta_{2}$ and the affine Weyl group elements $w_{\hat{1}}, w_{0}, w_{2}$, respectively.

We look next at the fusion of the fundamental representation with the representations on the border line $[0, n], 0<n<p-1$. These representations are counterparts of the conjugates of the symmetric representations and the intersection of their assumed shifted weight diagrams $\mathcal{G}_{[0, n]} \circ f$ with the fundamental 7 point diagram $\mathcal{G}_{f} \circ[0, n]$ (both defined by the "shifted" highest weight $f+[0, n]=[1, n])$ contains at most 3 points corresponding to the elements $\mathbf{1}, w_{121}$, and $w_{0121}$ acting on the weight $[1, n]$, see e.g. for $n=2$ the mirror counterpart of the diagram on fig. 2. The fusion of the representations on the other two $1^{\text {st }}$ leaf border lines $\left(\left[n_{1}, n_{2}\right], n_{2}=0\right.$, or $\left.n_{1}+n_{2}=p-1\right)$ are recovered by the $\mathbb{Z}_{3}$ symmetry using (5.1).

We next turn to the fusion of the fundamental representation $f$ with the representations on the second leaf starting with the point $[[1,1]]$. The two systems of algebraic equations corresponding to the roots $\beta_{\hat{\imath}}=\delta-\alpha_{i}, i=1,2$, exclude all but 3 of the representations in (4.2) expressed by the action of $w_{21}, w_{121}$, and $w_{0121}$. Similarly for representations on the border line $[[n, 1]], 1<n<p-1$, of the second alcove the solution of the additional system of equations corresponding to the root $\beta_{\hat{1}}=\delta-\alpha_{1}$ excludes the first two of the seven terms in (4.2). Once again we extend this result to the rest $2^{\text {nd }}$ leaf border lines using the $\mathbb{Z}_{3}$ symmetry.

The truncations described appear only on the borders of the double alcove (invariant under the symmetry (2.2)). Note that all representations in the r.h.s. of (4.2) belong to the admissible domain whenever the weight $\Lambda^{\prime}$ in the l.h.s is an interior point (i.e., the interior points are generic points) - this is easier to visualize on the "big" alcove, see fig 1, whose "border" lines accommodate all border points of the double alcove. - simply attach the 7 point diagram to the given highest weight.

In principle one should look further at the solutions of the remaining equations resulting from more complicated MFF vectors and check whether they lead to further truncations. E.g., in the case $[[n, 1]]$ we should consider also the singular vector corresponding to the root $\beta_{\hat{2}}=n \delta-\alpha_{2}$; apparently the (reduced to polynomials) form of this MFF 
singular vector gets increasingly complicated for large $n$. On the other hand it is natural to expect that if there are additional truncations they will appear for smaller $p$. So we have considered more thoroughly the smallest case $p=4$ where the MFF vectors are the simplest possible - the outcome is that there are no further truncations.

The result of this analysis is summarised in the following

\section{Proposition 2:}

For interior points on $P_{3, p}$ the $\mathrm{FR}$ read

$$
\begin{aligned}
& f \otimes\left[n_{1}, n_{2}\right]=\left[n_{1}+1, n_{2}\right] \oplus\left[n_{1}-1, n_{2}+1\right] \oplus\left[n_{1}, n_{2}-1\right] \oplus\left[n_{2}, p-1-n_{3}\right] \\
& \oplus\left[\left[n_{1}+1, n_{2}\right]\right] \oplus\left[\left[p-1-n_{3}, n_{1}+1\right]\right] \oplus\left[\left[n_{2}+1, p-n_{3}\right]\right] \\
& f \otimes\left[\left[n_{1}, n_{2}\right]\right]=\left[\left[n_{1}+1, n_{2}\right]\right] \oplus\left[\left[n_{1}-1, n_{2}+1\right]\right] \oplus\left[\left[n_{1}, n_{2}-1\right]\right] \oplus\left[\left[p+1-n_{3}, n_{1}\right]\right] \\
& \oplus\left[n_{1}, n_{2}-1\right] \oplus\left[p-n_{3}, n_{1}-1\right] \oplus\left[n_{2}-1, p+1-n_{3}\right]
\end{aligned}
$$

On the border lines of the two alcoves constituting $P_{3, p}$ the FR multiplicities read

$$
\begin{aligned}
f \otimes[0, n] & =[1, n] \oplus[0, n-1] \oplus[[1, n]], \quad 0<n<p-1 ; \quad f \otimes[0,0]=f ; \\
f \otimes[[n, 1]] & =[[n+1,1]] \oplus[[n-1,2]] \oplus[[p-n, n]] \oplus[n, 0] \oplus[p-n-1, n-1], \quad 1<n<p-1, \\
f \otimes[[1,1]] & =[[2,1]] \oplus[[p-1,1]] \oplus[1,0] ;
\end{aligned}
$$

the rest being determined by the $\mathbb{Z}_{3}$ symmetries (5.1).

Following the approach of [14], [15] we can look at the set of rules (5.2), (5.3) as defining for each $p$ the adjacency matrix $G_{a b}=N_{f a}^{b}$ of a fusion graph, the vertices of which correspond to the points of the corresponding double alcove $P_{3, p}$; we do not indicate explicitly the links of this graph. The complex conjugated representation $f^{*}$ provides the conjugated adjacency matrix $G_{a b}^{*}=N_{f^{*} a}^{b}$ describing the same graph with inverted orientation of the links; $G_{a b}^{*}=G_{b a}$. Unlike the known fusion graphs for integrable representations of $\widehat{s l}_{3}$ and their nondiagonal generalisations studied in [15] (see also [16]) the graphs described by (5.2), (5.3) are not 3-colourable - if we assign the usual triality $\tau(a)=n_{1}-n_{2}$ to the vertices $a=\left[n_{1}, n_{2}\right]$ and $a=\left[\left[n_{1}, n_{2}\right]\right]$ we will find nonvanishing matrix elements $G_{a b}$ for some $a, b$, such that $\tau(b)-\tau(a) \neq 1 \bmod 3$. Nevertheless these graphs share some of the basic features of their integrable counterparts, in particular the $\mathbb{Z}_{3}$ symmetry, replaced here by the pair (2.2) discussed above. The matrix $G$ is normal, $\left[G, G^{t}\right]=0$, and hence diagonalisable. Its normalised eigenvectors $\psi_{a}^{(\mu)}$

$$
\sum_{a} \psi_{a}^{(\mu)} \psi_{a}^{(\nu) *}=\delta_{\mu \nu}, \quad \sum_{\mu} \psi_{a}^{(\mu)} \psi_{c}^{(\mu) *}=\delta_{a c}
$$


labelled by some set $\{\mu\}$ of $p^{2}$ indices can be used to write down a formula for the general FR multiplicities $N_{a b}^{c}$ of the type first considered by Pasquier [14]

$$
N_{a b}^{c}=\sum_{\mu} \frac{\psi_{a}^{(\mu)} \psi_{b}^{(\mu)} \psi_{c}^{(\mu) *}}{\psi_{\mathbf{1}}^{(\mu)}} .
$$

The formula can be looked at as a generalisation of the Verlinde formula with the matrix $\psi_{a}^{(\mu)}$ replacing the (symmetric) modular matrix. In (5.5) the vector $\psi_{1}^{(\mu)}$, the (dual) PerronFrobenius vector, is required to have nonvanishing entries. In the simplest example of $p=4$ we have checked that there exists a choice of the eigenvector matrix $\psi_{a}^{(\mu)}$ consistent with this requirement (and furthermore $\psi_{\mathbf{1}}^{(\mu)}>0$ ) and such that (5.5) produces nonnegative integers for the matrix elements of all $N_{a}$ matrices, $\left(N_{a}\right)_{b}^{c}=N_{a b}^{c}$. The arbitrariness in determining the eigenvectors is caused by the presence of eigenvalues of $G$ of multiplicity greater than one. The eigenvalues $\chi_{a}(\mu)=\psi_{a}^{(\mu)} / \psi_{1}^{(\mu)}$ provide 1 - dimensional representations (" $q$ characters") of the algebra of $N_{a}$-matrices. In the $\operatorname{sl}(2)$ case the analogous to (5.5) formula (which is more explicit since there is a general expression for the matrices $\psi_{a}^{(\mu)}$ ) reproduces indeed the known [3] FR multiplicities at level $2 / p-2$. It should be stressed that the $1^{\text {st }}$ leaf representation $f$ and its adjoint $f^{*}$ do not exhaust the fundamental set of fields needed to generate a fusion ring. However the description of that set is not needed in formula (5.5), the latter providing the full information about the fusion rule. In writing (5.5) we have essentially assumed that the admissible reps fusion algebra is a " $\mathrm{C}$ - algebra" (from "Characters - algebra"), following the terminology in [17], i.e., an associative, commutative algebra over $\mathbb{C}$ with real structure constants, with a finite basis, an identity element, an involution (here the map of a weight to its adjoint) requiring some standard properties of the structure constants. The knowledge of the fundamental matrix $N_{f}$ specifies the algebra and allows to describe the common to all matrices $N_{a}$ eigenvector matrix $\psi_{a}^{(\mu)}$. The fact that the general formula (5.5) for the "C - algebra" structure constants gives nonnegative integers is highly nontrivial. Comparing with [15] (where such " $\mathrm{C}$ - algebras" were discussed and used), recall that the nonnegativity of the integers in the 1.h.s. of formulae analogous to (5.5) selects a subclass of the graphs related to modular invariants of the integrable models; a counterexample, where this nonnegativity cannot be achieved, is provided e.g., by the $E_{7}$ Dynkin diagram.

4 More precisely a formula analogous to the dual version of (5.5), obtained by a summation over the vertices, appeared in [14], producing in general noninteger structure constants $M_{\mu \lambda}^{\nu}$. 
Thus the knowledge of the fundamental fusion graph allows to determine in principle all FR multiplicities.

Remark: The graphs appearing in the study of the admissible representations and the structures they determine deserve further investigation. In particular it is yet unclear whether the "dual" version of (5.5), describing the structure constants of a dual "C algebra", has any importance. It would be also interesting to check whether the set $\{\mu\}$, can be interpreted as some "exponents" set in the sense of [16]. These questions have sense already for the case of admissible representations of $\widehat{s l}(2)$ at level $2 / p-2$ where the corresponding graphs (or their unfolded colourable ladder type counterparts) look rather simple.

The basic FR (5.2), (5.3) which we have derived are checked to admit another interpretation which generalises the corresponding fusion formula of [4], [5], [6] in the case of integrable representations. First a representation $\Lambda^{\prime \prime}$ appears in the fusion $f \otimes \Lambda^{\prime}$ only if it belongs to the intersection of the 7 points "shifted" weight diagram of the fundamental representation $\mathcal{G}_{f} \circ \Lambda^{\prime}$ with the double alcove $P_{3, p}$; if $\mathcal{G}_{f} \circ \Lambda^{\prime} \subset P_{3, p}$ all 7 points in the fusion survive. The truncations in (5.3) are precisely described by (intersections of) orbits of the KW affine Weyl group starting from a point on $\mathcal{G}_{f} \circ \Lambda^{\prime} \cap P_{3, p}$ and reaching points of $\mathcal{G}_{f} \circ \Lambda^{\prime}$ outside of $P_{3, p}$. Assigning (according to (4.2) ) multiplicity one to all points of $\mathcal{G}_{f}$ (or to its isomorphic image $\mathcal{G}_{f} \circ \Lambda^{\prime}$ ), the points on any orbit contribute with a sign $\operatorname{det}(w$ ) determined by the corresponding element $w=w_{\beta}$; here $\operatorname{det}(w)$ is 1 or -1 if the number of elementary reflections of $W$ representing $w_{\beta}$ is even or odd. To visualize these orbits draw the big alcove for a given $p$ as in fig. 1. and attach on it the shifted diagram $\mathcal{G}_{f} \circ \Lambda^{\prime}-$ the shifted highest weight itself can be outside of the alcove.

Another example of this truncation mechanism is provided by the fusions of $[1,1]$. Taking a sufficiently large $\Lambda^{\prime}$ in the fusion $[1,1] \otimes \Lambda^{\prime}$ - i.e., $\Lambda^{\prime}$ and $p$ such that $\mathcal{G}_{[1,1]}{ }^{\circ}$ $\Lambda^{\prime} \subset P_{3, p}$, so that no truncations occur, the FR multiplicities coincide with the weight multiplicities of $[1,1]$ and can be computed from the general formula (5.5). Alternatively the same weight multiplicities can be recovered (and that is how originally we obtained the values indicated on fig. 4), by the above mechanism of truncation along orbits of the KW group, given the FR multiplicities for "smaller" $\Lambda^{\prime}$ and $p$. 


\section{6. "Verma modules" and weight diagrams. The role of $\mathrm{KW}$ affine Weyl group as a "truncating" group.}

We shall now formulate in a more concise general form the analog of the FR multiplicities formula of [4], [5], [6] for integrable representations "inverting" the argumentation of [6]. As a motivation for what follows recall that the $\operatorname{sl}(3)$ finite dimensional representations, equivalently their supports, i.e., the weight diagrams $\Gamma_{\lambda}$, can be resolved via the action of $\bar{W}$ in terms of $\operatorname{sl}(3)$ Verma modules.

First we introduce some more notation. Recall that $\bar{\alpha}_{0}=-\alpha_{3}, \bar{\alpha}_{i}=\alpha_{i}, i=1,2$. Given an affine root $\alpha$ among the subset $\left\{\alpha_{i}, \alpha_{\hat{\imath}}=\alpha_{0}+\alpha_{3}-\alpha_{i}, i=0,1,2\right\}$ there is a projection $\bar{\alpha}$ in the set of $\operatorname{sl}(3)$ roots. Accordingly $w_{\alpha} \in W$ projects to $\bar{w}_{\alpha}=w_{\bar{\alpha}} \in \bar{W}$. Vice versa given a $\operatorname{sl}(3)$ root and a corresponding reflection there is an unique affine root among the above subset and a corresponding reflection in $W$. Similarly for any set of $\operatorname{sl}(3) \operatorname{roots} w(\bar{S}), w \in \bar{W}, \bar{S}=\left\{\alpha_{1}, \alpha_{2}\right\}$, there is a set $S_{w}$ comprised of a pair of affine roots such that it projects to $w(\bar{S})$. For even (odd) length $w \in \bar{W}$ the sets $S_{w}$ consist of roots in $\left\{\alpha_{i}\right\} \quad\left(\left\{\alpha_{\hat{\imath}}\right\}\right) i=0,1,2$, respectively. Let $Q^{w}=\oplus_{\alpha \in w(\bar{S})} \mathbb{Z}_{+} \alpha$ for $w \in \bar{W}$. We recover $Q^{+}$for $w=i d$. For $w \in \bar{W}$ of even length let $T(w)$ be a group isomorphic to $\bar{W}$ generated by the affine reflections labelled by the roots in $S_{w}: T(i d) \equiv \bar{W}, T\left(w_{21}\right)$ is generated by $\left\{w_{0}, w_{1}\right\}$, etc. For odd length $w \in \bar{W}$ the set $T(w)$ (which also projects to $\bar{W})$ is defined as $\left\{\mathbf{1}, w_{\hat{\imath}}, w_{\hat{\jmath}}, w_{j k}, w_{i k}, w_{k}\right\}, \hat{k} \neq \hat{\imath}, \hat{\jmath}$, if $S_{w}=\left\{\alpha_{\hat{\imath}}, \alpha_{\hat{\jmath}}\right\}, i, j=0,1,2, i \neq j$. E.g., $T\left(w_{3}\right)=\left\{\mathbf{1}, w_{\hat{2}}, w_{\hat{1}}, w_{10}, w_{20}, w_{0}\right\}$.

Recalling that the affine Weyl group $W$ is a semidirect product of the horizontal (finite) Weyl group $\bar{W}$ and the root lattice $Q$, one can view the affine Weyl group graph $\mathcal{W}$ introduced above as the translations of the basic hexagon, the graph of $\bar{W}$, by $\kappa Q$, i.e., action on the six vertices of the basic hexagon by powers of $w_{i \hat{\imath}}$ or $w_{\hat{\imath} i}, i=0,1,2$. More generally for $w \in \bar{W}$ define

$$
\mathcal{W}_{\Lambda}^{w}=\cup_{w^{\prime} \in T(w)}\left(w^{\prime} \cdot \Lambda+\kappa Q^{\overline{w^{\prime}} w}\right), \quad w \in \bar{W}
$$

In particular $\mathcal{W}_{\Lambda}^{\text {id }}$ coincides with $\mathcal{W}_{\Lambda}^{+}$from above (see figs. 2,5 ), while the subgraph $\mathcal{W}_{\Lambda}^{-}:=$ $\mathcal{W}_{\Lambda}^{w_{3}}$ is illustrated on fig. 6 .

By analogy with the $s l(3)$ case where the set $\lambda-Q^{+}$describes the support of the Verma module of highest weight $\lambda$ we shall refer to $\mathcal{W}_{\Lambda}^{w}$ as to "Verma modules" of highest weight $\Lambda$. The range of $\Lambda$ is not confined to the admissible domain (2.1) and accordingly we can drop at this point the requirement of rationality of the parameter $\kappa$. 
In the case when the affine Verma module of highest weight $\Lambda=-\lambda \kappa$ or $\Lambda=w_{3} \cdot(-\lambda \kappa)$ contains by the Kac-Kazhdan theorem a singular vector of weight $w_{\beta_{i}} \cdot \Lambda$ or $w_{\beta_{\hat{\imath}}} \cdot \Lambda, i=1,2$ (i.e., $\lambda$ is integral dominant, $\lambda \in P_{+}$, or strictly integral dominant, $\lambda \in P_{++}$) then this weight belongs to the graph $\mathcal{W}_{\Lambda}^{+}$or $\mathcal{W}_{\Lambda}^{-}$respectively. We can view these weights as the highest weights of "Verma submodules" $\mathcal{W}_{w_{\beta_{i}} \cdot \Lambda}^{w_{i}}$ and $\mathcal{W}_{w_{\beta_{\hat{\imath}}} \cdot \Lambda}^{w_{i 3}}$, respectively. One can extend this to the other four types of "Verma modules" with proper infinite range of $\Lambda$ dropping the upper bounds of the alcoves. Thus we have six different analogs of the $\operatorname{sl}(3)$ reducible Verma modules (of integral dominant highest weights), the singular vectors of which are governed by the corresponding KW horizontal group $\bar{W}^{\lambda}$.

One can think of the "Verma modules" introduced here as comprising some "extended" in the sense of [10] states given by compositions of noninteger powers of the three lowering generators $E^{-\alpha_{i}}, i=0,1,2$, of $\widehat{s l}(3)$ - these powers are dictated by the (shifted) affine Weyl reflections indicated on the graph $\mathcal{W}$, in particular any path from the origin to a "singular vector" corresponds to a MFF expression for the "true" singular vector of the affine algebra Verma module. This intuitive picture is quite precise in the $\widehat{s l}(2)$ case where all states of the corresponding "extended" Verma modules have multiplicity one. Then these modules are seen to be isomorphic to Verma modules of the superalgebra osp $(1 \mid 2)$; the two sublattices of the corresponding graphs, associated with the two elements of the $s l(2)$ Weyl group, correspond to even or odd submodules with respect to $\operatorname{sl}(2)$.

The problem of multiplicities however makes doubtful the usefulness of such "extended Verma modules" interpretation in our case and what replaces the above superalgebra is yet to be seen. Instead we follow further the analogy with the $\operatorname{sl}(3)$ case and we assign multiplicities $K_{\mu}^{\Lambda}$ to the weights $\mu$ of a general "Verma module" of highest weight $\Lambda$, starting from a multiplicity 1 assigned to the points on the border rays, and with each step inward increasing by 1 the multiplicity at the points of the subsequent pair of lines parallel to them (going through diagonals of the honeycomb hexagons) ; see fig. 5,6 where this assignment is depicted. Not to overburden the notation we shall not indicate explicitly the dependence of $K_{\mu}^{\Lambda}$ on the type of module. There are simple formulae (to be presented elsewhere) for the generating functions of the weight multiplicities assigned to any of the 6 sublattices in (6.1) which generalise the Kostant generating function for $\operatorname{sl}(3)$ Verma modules.

Let $\Lambda=-\lambda \kappa$, or $\Lambda=w_{3} \cdot(-\lambda \kappa)$, with $\lambda \in P_{+}$, or $\lambda \in P_{++}$, respectively. We define now "weight diagram" multiplicities $m_{\mu}^{\Lambda}$ according to

$$
m_{\mu}^{\Lambda}=\sum_{w \in \bar{W}^{\lambda}} \operatorname{det}(w) K_{\mu}^{w \cdot \Lambda}, \quad \mu \in \mathcal{W}_{\Lambda}^{ \pm},
$$


and we shall refer to the (finite) collection of points $\{\mu\}$ with nonzero $m_{\mu}^{\Lambda}$ as "weight diagram" of $\Lambda$ to be denoted $\mathcal{G}_{\Lambda}$. The proof that this definition has sense, i.e., that the numbers in the l.h.s. of (6.2) are nonnegative integers, extends the corresponding argument for the weight diagrams of the finite dimensional representations of $\operatorname{sl}(3)$ and will appear elsewhere. The previously discussed examples are easily checked to fit the definition - in fact we have arrived at the Verma module multiplicities $K_{\mu}^{\Lambda}$ "inverting" (6.2), i.e., solving it for small values of $K_{\mu}^{\Lambda}$ with the l.h.s. provided by the examples. An example of a weight diagram of the second kind (i.e., of highest weight $\Lambda=w_{3} \cdot(-\lambda \kappa), \lambda \in P_{++}$) is provided by the 4-point diagram of the representation $h=[[1,1]]$ - the "Verma modules resolution" formula (6.2) for this case is illustrated in fig. 7, while figs. 2,3 illustrate (6.2) for $\Lambda=[1,0]$ and $\Lambda=[2,0]$. On all these figures the positions of the singular vectors (the two fundamental ones corresponding to the simple reflections in $\bar{W}^{\lambda}$ and the ones corresponding to compositions of such reflections) are indicated by $\star$.

The two types of diagrams $\mathcal{G}_{\Lambda}$ are illustrated furthermore on figs. 8,9. by two generic examples. View the vertices of $\mathcal{G}_{\Lambda}$ as lying on a set of "concentric" "hexagons" (which could degenerate into triangles); they are drawn through diagonals of the honeycomb elementary hexagons. The points on the outmost hexagon have multiplicity 1, and with every step inward the multiplicity increases by one if the layers are hexagons or stays constant once the layers degenerate into triangles. This is the direct generalisation of the rule for the $s l(3)$ weight diagram $\Gamma_{\lambda}$ multiplicities. In general the intersection of an weight diagram with any of the 6 lattices in (6.1) (for fixed $w=\mathbf{1}$, or $w=w_{3}$ ) decomposes into a collection of weight diagrams of $s l(3)$ irreducible representations.

The same pictures admit another interpretation which may serve as an alternative definition leading to (6.2): refining by 3 the lattice $Q \kappa$ (i.e., $p \rightarrow 3 p$ ) and scaling by 3 the weights $\lambda$ we can identify the two types of diagrams of highest weight $\Lambda$ with standard $\operatorname{sl}(3)$ weight diagrams with some points removed. Namely we map the highest weight $\Lambda=-\lambda \kappa$ $\left(\lambda \in P_{+}\right)$to $i(\Lambda):=3 \lambda$, and $\Lambda=w_{3} \cdot(-\lambda \kappa)\left(\lambda \in P_{++}\right)$to $i\left(w_{3} \cdot(-\lambda \kappa)\right)=3 \lambda-2 \rho$, resp. The points removed from the $s l(3)$ weight diagrams originate from the centers of the honeycomb hexagons shaping $\mathcal{G}_{\Lambda}$, i.e., having at least $3(2)$ common links with $\mathcal{G}_{\Lambda}$, see figs. 8,9 , where these points are indicated by squares. The weight multiplicities defined in (6.2) coincide with the $s l(3)$ weight multiplicities of the corresponding surviving points.

The same embedding applies to the supports of the Verma modules. The $s l(3)$ counterparts of the weights $\mu \in \mathcal{W}_{\Lambda}^{ \pm}$are recovered from the highest weight $\Lambda$. More explicitly, the same definition of the map $i$ applies to the points $\mu$ on the first, $w^{\prime}=1$ lattice in (6.1), 
$w=\mathbf{1}$, containing the highest weight. The rest are recovered from such points. Namely for $\Lambda=-\lambda \kappa$ and $\mu \in \Lambda+Q^{+} \kappa$, we have $i(w \cdot \mu)=i(\mu)+w^{-1} \cdot(0,0)$, for $w \in \bar{W}$. The points removed from the support $3 \lambda-Q^{+}$of the $s l(3)$ Verma module of highest weight $3 \lambda$ (not representing images of $\mathcal{W}_{\Lambda}^{+}$) belong to the subset $\cup_{\alpha>0}\left(3 \lambda-3 Q^{+}-(3-\langle\alpha, \rho\rangle) \alpha\right)$. This rule can be also used to recover the images of the "Verma modules" of highest weight $w_{3} \cdot(-\lambda \kappa)$, representing each point of $\mathcal{W}_{\Lambda}^{-}$as $w \cdot \mu$, with $w \in \bar{W}$ and some $\mu \in-\lambda \kappa+Q^{+} \kappa$. The definition (6.2) of the weight diagram multiplicities and the mapping into $s l(3)$ representations extend to the other 4 of the 6 different types of "Verma modules" (6.1), taken with a proper choice of the simple root system generating $\bar{W}^{\lambda}$; we omit here the details. Apparently this map preserves (up to permutation) the positions of the singular vectors, e.g., $i\left(w_{\beta_{j}} \cdot \Lambda\right)=w_{j} \cdot i(\Lambda), j=1,2, i\left(w_{\beta_{1}} w_{\beta_{2}} \cdot \Lambda\right)=w_{21} \cdot i(\Lambda)$, etc., i.e. the action of the $\mathrm{KW}$ groups is "converted" under this map into ordinary Weyl group action. This together with the conservation of the multiplicities $K_{\mu}^{\Lambda}=\bar{K}_{i(\mu)}^{i(\Lambda)}$, the latter denoting the multiplicity of the weight $i(\mu)$ in the $s l(3)$ Verma module of highest weight $i(\Lambda)$, converts (6.2) into the standard Verma modules resolution formula for $s l(3)$ taken for a subset of weights.

Note that if $\Lambda$ is in addition an admissible highest weight at level $3 / p-3$ the above defined weight $i(\Lambda)$ is an integrable highest weight at level $3 p-3$.

Because of the trivial triality of the $s l(3)$ counterparts (their weights lying on the $s l(3)$ root lattice $Q$ ) all diagrams of both types contain a "middle" point $\Lambda_{0}$ mapped to the $\operatorname{sl}(3)$ weight $(0,0)$. It is given by $\Lambda_{0}=\sigma^{-\tau(\Lambda)}([0,0])$, where we recall that $\tau(\Lambda)=n_{1}-n_{2} \bmod$ 3 for $\Lambda=\left[n_{1}, n_{2}\right]$ or $\Lambda=\left[\left[n_{1}, n_{2}\right]\right] ; \Lambda_{0}$ has a (maximal) multiplicity $3 \min \left(n_{1}, n_{2}\right)+1$ and $3 \min \left(n_{1}, n_{2}\right)-1$, respectively.

We have already discussed the "shifted weight diagram" $\mathcal{G}_{\Lambda} \circ \Lambda^{\prime}$ assigned to a highest weight $\Lambda$ sitting on the first leaf of $P_{3, p}$. In general for $\Lambda, \Lambda^{\prime} \in P_{3, p}$ the shifted weight diagram $\mathcal{G}_{\Lambda} \circ \Lambda^{\prime}$ is a diagram isomorphic to $\mathcal{G}_{\Lambda}, \mathcal{G}_{\Lambda} \ni \mu=w \cdot \Lambda \mapsto w \cdot\left(\Lambda \circ \Lambda^{\prime}\right) \in \mathcal{G}_{\Lambda} \circ \Lambda^{\prime}$, $w \in W$, with a reference point (shifted highest weight) $\Lambda \circ \Lambda^{\prime}:=\Lambda+w\left(\Lambda^{\prime}\right)$ with $w=\mathbf{1}$ $\left(w=w_{3}\right)$ if $\Lambda$ is on the first (second) leaf of $P_{3, p}$, respectively. The weight multiplicities of $\mathcal{G}_{\Lambda} \circ \Lambda^{\prime}$ are given by

$$
m_{w \cdot\left(\Lambda \circ \Lambda^{\prime}\right)}^{\Lambda ; \Lambda^{\prime}}:=m_{w \cdot \Lambda}^{\Lambda}, \quad w \in W,
$$

where the r.h.s. is determined from (6.2). Alternatively the shifted weight diagram can be recovered from its middle point $\sigma^{-\tau(\Lambda)}\left(\Lambda^{\prime}\right)$; recall that the definition of the generating element $\sigma\left(\Lambda^{\prime}\right)$ in (2.2) depends on the type of $\Lambda^{\prime}$.

The mapping into $\operatorname{sl}(3)$ diagrams preserving weight multiplicities can be extended to the shifted diagrams identifying in particular the $\operatorname{sl}(3)$ shifted highest weight with 
$i(\Lambda)+i\left(\Lambda^{\prime}\right)$. Note that if both $\Lambda, \Lambda^{\prime}$ are points on the $2^{\text {nd }}$ leaf this weight originates from a center of a (boundary) hexagon on the shifted weight diagram $\mathcal{G}_{\Lambda} \circ \Lambda^{\prime}$, i.e, from a point beyond $\mathcal{G}_{\Lambda} \circ \Lambda^{\prime}$ itself. The image of the shifted highest weight coincides with $w(i(\Lambda))+i\left(\Lambda^{\prime}\right)$ (a point on the $s l(3)$ shifted weight diagram), where $w=\mathbf{1}\left(w=w_{3}\right)$ if $\Lambda^{\prime}$ is on the first (second) leaf, respectively.

We are now at a position to formulate the analog of the FR multiplicities formula in [4], [5], [6].

Proposition 3. The FR multiplicities of (a triple of) admissible weights on the double alcove $P_{3, p}$ are given by the formula

$$
N_{\Lambda \Lambda^{\prime}}^{\Lambda^{\prime \prime}}=\sum_{w \in W^{\lambda^{\prime \prime}}} \operatorname{det}(w) m_{w \cdot \Lambda^{\prime \prime}}^{\Lambda ; \Lambda^{\prime}}
$$

At present this statement is rather a conjecture supported by a lot of empirical data, in particular it is established if one of the three highest weights in (6.4) coincides with the fundamental weights $f$ or $f^{*}$. It has been also thoroughly checked for small values of $p \geq 4$; (6.4) extends to the degenerated case $p=2, p^{\prime}=3$ excluded so far.

An example illustrating (6.4) with $\Lambda$ sitting on the second leaf of the alcove is provided by the representation $h=[[1,1]]$. Its weight diagram appeared in fig. 7 . and its fusions read国

$$
h \otimes \Lambda^{\prime}=2 \Lambda^{\prime} \oplus w_{1} \cdot \Lambda^{\prime} \oplus w_{2} \cdot \Lambda^{\prime} \oplus w_{0} \cdot \Lambda^{\prime}
$$

for any representations $\Lambda^{\prime}$ on the second leaf and for generic (i.e., not on the border lines of the alcove) representations $\Lambda^{\prime}$ on the first leaf. For border representations on the first leaf (6.5) reduces to three multiplicity one terms (or to one such term if $\Lambda^{\prime}=\sigma^{l}(\mathbf{1})$ ). Apparently the latter truncation is a result of a 2-points orbit leading to $m_{\Lambda^{\prime}}-m_{w_{i} \cdot \Lambda^{\prime}}=2-1=1$ for $\Lambda^{\prime}$ such that $w_{i} \cdot \Lambda^{\prime} \notin P_{3, p}$, for some (one) $i=0,1,2$ or to a 3 -points orbit for $\Lambda^{\prime}=\sigma^{l}(\mathbf{1})$, in which case $w_{i} \cdot \Lambda^{\prime} \notin P_{3, p}$, for at least two $i=0,1,2$. The example $\Lambda^{\prime}=\sigma^{2}(\mathbf{1})$ is illustrated on fig. 10 .

For another example see fig. 11 and the Appendix. Note that unlike the integrable case there are no analogs here of " $q$-dim $=0$ " representations since the walls of the admissible alcove and its images by the action of the KW group do not support (being on the lattice dual to the graph $\mathcal{W}$ ) weights of "classical" representations.

5 This simplest representative of the $2^{\text {nd }}$ leaf admissible representations has to be included along with $\left\{f, f^{*}\right\}$ in the fundamental set generating the fusion ring. 
The nonnegativity of the FR multiplicities in (6.4) is ensured in general by the fact that the r.h.s of (6.4) can be expressed in terms of $s l(3)$ weight diagram multiplicities and the ordinary affine Weyl group $W$ adapting the map $i$ discussed above. One recovers in this way the r.h.s. of the formula in [4], [0], [6] for the FR multiplicities $N_{i(\Lambda) i\left(\Lambda^{\prime}\right)}^{i\left(\Lambda^{\prime \prime}\right)}(3 p)$ for particular triples of triality zero highest weights in an integrable theory at level $3 p-3$. (Recall that the Verlinde multiplicities are $\mathbb{Z}_{3}$ graded.) The details will be presented elsewhere, here we only illustrate this statement with an example depicted on fig. 12, see the Appendix for more details. The essential property used is that the (shifted action) Weyl group orbit of a "removed" point contains only "removed" points. This reformulation of (6.4) is a generalisation of the same type of relation between admissible and integrable representations FR multiplicities in the case of $\widehat{s l}(2)$ pointed out in (4.1) of [18]; in particular we expect that the general fusion rules for $p^{\prime} \geq 3$ will admit a similar factorised form in terms of fusion multiplicities for integrable representations at levels $p^{\prime}-3$ and $3 p-3$, see Appendix.

The formula (6.4) admits as in [6] a reformulation in terms of the analogs $m_{\Lambda \Lambda^{\prime}}^{\Lambda^{\prime \prime}}$ of the $s l(3)$ tensor product multiplicities. It applies to weights $\Lambda=w \cdot(-\lambda \kappa), w \in \bar{W}, \lambda \in P_{+}^{w}$, where $P_{+}^{1}=P_{+}, P_{+}^{w_{3}}=P_{++}$, and $P_{+}^{w_{i}} \equiv P_{+}^{w_{i 3}}=\left\{\lambda \in P_{+},\left\langle\lambda, \alpha_{i}\right\rangle \neq 0\right\}, i=1,2$. In particular for $\Lambda, \Lambda^{\prime}$ of the type $w \cdot(-\lambda \kappa), w=\mathbf{1}, w_{3}$, the multiplicities $m_{\Lambda \Lambda^{\prime}}^{\Lambda^{\prime \prime}}$ are defined as in (6.4), with the affine $\mathrm{KW}$ group $W^{\lambda^{\prime \prime}}$ replaced by its horizontal counterpart $\bar{W}^{\lambda^{\prime \prime}}$, generated by $w_{\gamma_{i}}, \gamma_{i}:=\left\langle\lambda^{\prime \prime}, \alpha_{i}\right\rangle \delta+w\left(\alpha_{i}\right), i=1,2$, for $\Lambda=w \cdot\left(-\lambda^{\prime \prime} \kappa\right), w \in \bar{W}$. It has been checked on numerous examples that the latter definition of $m_{\Lambda \Lambda^{\prime}}^{\Lambda^{\prime \prime}}$ makes sense, in particular leading to the conservation of the "classical" dimensions computed from the weight diagrams. We conclude with some of the simplest examples

$$
\begin{aligned}
f \otimes f^{*} & =\mathbf{1} \oplus h \oplus[1,1], \quad(7 \times 7=1+5+43), \\
f \otimes f & =f^{*} \oplus[2,0] \oplus w_{1} \cdot[2,0], \quad(7 \times 7=7+19+23), \\
h \otimes h & =\mathbf{1} \oplus 2 h \oplus w_{1} \cdot h \oplus w_{2} \cdot h, \quad(5 \times 5=1+2.5+7+7), \\
h \otimes f & =f \oplus w_{1} \cdot f \oplus[[2,1]], \quad(5 \times 7=7+5+23) .
\end{aligned}
$$

This is a strong indication that our weight diagrams are the supports of finite dimensional representations of a "hidden" algebra. Its $q$-version at roots of unity would provide eventually a truncated tensor product equivalent to the fusion rules. 


\section{Summary and conclusions.}

There are several different descriptions and derivations of the fusion rules at integer level. No one of them is easily extendable to the case of fractional level. Instead we have used a combination of several methods - neither of them is completely rigorous or thorough at present.

We started with the method which directly generalises the approach exploited by [3] in the derivation of the admissible representations fusion rules in the $\widehat{s l}(2)$ case. This is the singular vectors decoupling method applied to 3-point functions which works for fusions resulting in trivial (zero or one) fusion multiplicities. In particular it enabled us to determine the fusion of the (fractional) fundamental representations $f=[1,0]$ and $f^{*}=[0,1]$ with an arbitrary generic admissible representation $\Lambda^{\prime}$. The notions of weight diagram and shifted weight diagram described by a set of words in the affine Weyl group naturally arise as generalisations of the $s l(3)$ counterparts.

Unlike the $s l(2)$ case the decoupling method is technically rather involved due to the complexity of the general MFF singular vectors and presumably it has to be further elaborated in order to treat cases with nontrivial multiplicities. Instead we have followed a strategy influenced to some extent by the study [14], [15] of graphs (generalising the ADE Dynkin diagrams) related to nondiagonal modular invariants of the integrable WZNW theories. Namely selecting and analysing a subset of the decoupling systems of equations, corresponding to representations on the border paths of the admissible alcove, we have determined (under some additional assumptions) for $p^{\prime}=3$ and any $p \geq 4$ a fundamental fusion graph described by the fusion matrix $N_{f}$. This allowed us to write a formula, borrowed from [14], [15], for the general FR multiplicities at level $3 / p-3$. It generalises the Verlinde formula in which the symmetric modular matrix is replaced by the eigenvectors matrix $\psi_{a}^{(\mu)}$ of $N_{f}$. It is highly nontrivial that a formula of this kind gives nonnegative integers - we have no general proof of this fact checked for small values of $p$.

Knowing the matrix $N_{f}$ one can determine in principle for any $p$ its eigenvectors - yet the proposed Pasquier - Verlinde type formula (5.5) is still not very explicit in view of the absence so far of a general analytic formula for $\psi_{a}^{(\mu)}$. So our next step was to look for an alternative formula for FR multiplicities, generalising our old work in [6], see also [4], [5]. While the previous approach can be looked at as related to a resolution of the irreducible admissible representations in terms of a kind of generalised reducible "Fock modules" (since the differential operators realisation of the generators of $\widehat{s l}(3)$ is equivalent to a generalised 
free field bosonic realisation) the formula (6.4) described in section 6 rather relies on the idea of "Verma modules" resolution.

We recall that the starting point in [6] was the standard Weyl formula for the multiplicities of irreps in tensor products of $\operatorname{sl}(n)$ finite dimensional representations. This formula involves the weight multiplicities of $\operatorname{sl}(n)$ finite dimensional representations, i.e., their weight diagrams, which can be recovered by resolution of $\operatorname{sl}(n)$ Verma modules. So it was natural to try to interpret similarly the generalised weight diagrams we have encountered in section 4 - formula $(6.2)$ is precisely of this type with the Weyl group $\bar{W}$ replaced by the horizontal KW group $\bar{W}^{\lambda}$.

The hard part in this alternative approach is the absence initially of an obvious candidate for the finite dimensional algebra whose representation theory matches the structures introduced in section 6 . Instead we have described the "Verma modules" by their supports, i.e., the set of weights and their multiplicities. Our final step was as in [6] to "deform" the classical formulae replacing the horizontal Weyl group with its affine analog, in our case the affine KW group $W^{\lambda}$. While in [6] we have been guided at this step by the representation theory of the deformed algebra $U_{q}(s l(n))$ for $q$ - a root of unity, here once again we lack so far the $q$ - counterpart of the hidden algebra - rather the consistency of (6.4) with the alternative approaches suggests the existence of such deformation.

The emerging finite dimensional (graded) algebra (and its $q$ - counterpart) behind the series $\kappa=3 / p$ of $A_{2}^{(1)}$ admissible representations is the most interesting outcome of this work. It might be possible to recover this algebra (containing $\operatorname{sl}(3)$ as a subalgebra) from the supports of the (reducible) Verma modules introduced above. In fact there is some evidence that the algebra is encoded by the 43 - dimensional representation [1, 1], the fractional "adjoint" representation. In particular the map $i$ discussed in section 6 provides a natural way of introducing a root system related to the weight diagram of this representation. While a subset of these roots sits on the $\operatorname{sl}(3)$ weight lattice $P$ (which contains the $\operatorname{sl}(3)$ root lattice $Q)$, there are "fractional" roots beyond it.

Another remaining related problem is the description of the characters of the finite dimensional representations of this algebra and their $q$-version, i.e., the derivation of an explicit formula for the eigenvectors matrix $\psi_{a}^{(\mu)}$. This would give more substance to the analog (5.5) of the Verlinde formula and would eventually allow to prove its equivalence to (6.4), so far checked only on examples. 
Starting from an explicit finite dimensional algebra may simplify also a more abstract derivation of the $\widehat{s l}(3)$ admissible representations fusion rules as well as their generalisation to $\widehat{s l}(n)$.

These questions are under investigation.

Let us finally mention that the problem of deriving the admissible representations fusion rules might be relevant for the analogous problem for representations of $W$-algebras obtained by quantum (non principal) Drinfeld-Sokolov reduction from the affine algebras - see the analogous reduction of Verma modules singular vectors in [11].

\section{Acknowledgements}

We would like to thank V. Dobrev, V. Molotkov, Tch. Palev, I. Penkov, V. Schomerus and V. Tolstoy for useful discussions. A.Ch.G. and V.B.P. acknowledge the hospitality and the support of INFN, Trieste. A.Ch.G. acknowledges the support of the Alexander von Humboldt Foundation. This paper was partially supported by the Bulgarian Foundation for Fundamental Research under contract $\Phi-404-94$.

\section{Appendix A. Examples}

On figs. 11 we illustrate the fusion

$$
[[2,1]] \otimes[0,1]=[[2,2]] \oplus[1,1] \oplus[[1, a]] \oplus[[1,1]] \oplus[a, 1], \quad a=p-2
$$

On the first fig. 11a we depict the weight diagram $\mathcal{G}_{\Lambda}$ for $\Lambda=[[2,1]]$; the multiplicities are indicated in circles. On fig. $11 \mathrm{~b}$ we depict the same diagram but shifted by $\Lambda^{\prime}=[0,1]$, i.e., the shifted diagram $\mathcal{G}_{\Lambda} \circ \Lambda^{\prime}$ having $\sigma^{2}\left(\Lambda^{\prime}\right)=[1, a]$ at the middle point; for short $a=p-2$, $b=p-3$. The shifted diagram is situated on the graph $\mathcal{W}$ viewed as a weight lattice. All weights sitting outside the addmissible alcove are indicated by (??) and the weights of the first and the second leaf are indicated by circles and boxes, respectively. Reflecting in the dotted lines (the walls of the alcove) gives the truncations. The weights $[[a, 1]]$ and $[1, a]$ get their multiplicities truncated to $0=2-2$; [b,0] to $0=1-1 ;[0,0]$ to $0=2-1-1$; $[[1,1]]$ and $[1,1]$ to $1=2-1$.

On fig. 12 we illustrate the relation between the fusion rules of admissible representations at level $3 / p-3$ and of the integrable representations at level $3 p-3$. We compare the fusions $[1,1] \otimes[1,0]$ and $(3,3) \otimes(3,0)$ choosing $p=4$. 
The shifted weight diagram $\mathcal{G}_{[1,1]} \circ[1,0]$ is mapped into the weight diagram $\Gamma_{(3,3)}+$ $(3,0)+\rho$, the shifted highest weight $\mu=[1,1]+[1,0]=[2,1]$ is identified on the picture with the $s l(3)$ shifted highest weight $(6,3)+\rho=(7,4)$. The reason we shift here in addition the integrable weight with $\rho$ is that following the tradition we describe the fusion of integrable representations geometrically by ordinary, nonshifted Weyl group action, but acting on the shifted by $\rho$ weights; in the final result all surviving weights are shifted back with $-\rho$. The boundary triangle enclosing the admissible alcove $P_{3,4}$ is mapped into the boundary lines of the $\widehat{s l}(3)$ integrable alcove $P_{+}^{12}$. The origin $[0,0]$ of $P_{3,4}$ corresponds to the $s l(3)$ weight $(10,1)$, i.e., the corner point $\sigma((1,1))$ of the alcove $P_{++}^{12}$. (For $p=5$ it would have corresponded to $(1,13)=\sigma^{*}((1,1))$ in $P_{++}^{15}$.)

The "removed" weights sitting at the centers of the elementary hexagons are indicated by squares. Such are in particular the weights $\lambda^{\prime \prime}, \lambda^{\prime \prime}+\rho=(7,4)-2 \alpha_{1},(7,4)-2 \alpha_{3}-$ $\alpha_{1},(7,4)-\alpha_{3}$ appearing with multiplicity one in the product $(3,3) \otimes(3,0)$. The remaining 7 weights $\lambda^{\prime \prime}$ in this product, $\lambda^{\prime \prime}+\rho=(7,4),(5,5),(1,7),(4,4),(5,2),(4,1),(2,5)$, have admissible counterparts all of multiplicity one, recovered by the inverse map $i^{-1}$. E.g., $i([2,1])=(6,3)$, and hence $i^{-1}((6,3))=[2,1]$. Similarly $i^{-1}((3,0))=[1,0]=w_{0 \hat{0}} \cdot[2,1]$, $i^{-1}((0,6))=[0,2]=w_{\hat{1} 1} \cdot[2,1]$. Furthermore $i\left(w_{1} \cdot[2,1]\right)=i([2,1])-\alpha_{1}=(6,3)-\alpha_{1}=$ $(4,4)$, hence $i^{-1}((4,4))=w_{1} \cdot[2,1]=[[1,2]]$. Similarly $i^{-1}((4,1))=i^{-1}\left((6,3)-2 \alpha_{3}\right)=$ $w_{3} \cdot[2,1]=[[2,1]]$, and $i^{-1}((1,4))=w_{2} \cdot[0,2]=w_{021} \cdot[2,1]=[[2,2]], i^{-1}((3,3))=$ $i^{-1}\left((6,3)-\alpha_{1}-\alpha_{3}\right)=w_{21} \cdot[2,1]=[1,1]$.

The final result can be checked to coincide with what is prescribed by (4.2). It can be cast into the form

$$
\Lambda_{1} \otimes \Lambda_{2}=\sum_{\lambda_{3}}^{\prime} N_{3 \lambda_{1} 3 \lambda_{2}}^{\lambda_{3}}(3 p) w^{\left(\lambda_{3}\right)} \cdot\left(\Lambda_{1}+\Lambda_{2}\right),
$$

where $w^{\left(\lambda_{3}\right)} \in W$ is determined from $i\left(w^{\left(\lambda_{3}\right)} \cdot\left(\Lambda_{1}+\Lambda_{2}\right)\right)=\lambda_{3}$ as described above, the multiplicity in the r.h.s. is the integrable FR multiplicity at level $3 p-3$ and the prime in the sum indicates that all "removed" points are excluded.

By analogy with (4.1) of [11] the expected generalisation for arbitrary $p^{\prime}$ and admissible ( $1^{\text {st }}$ leaf) highest weights $\Lambda_{i}=\lambda_{i}^{\prime}-\lambda_{i} \kappa, i=1,2$ reads

$$
\Lambda_{1} \otimes \Lambda_{2}=\sum_{\lambda_{3}^{\prime}} \sum_{\lambda_{3}}^{\prime} N_{\lambda_{1}^{\prime}, \lambda_{2}^{\prime}}^{\lambda_{3}^{\prime}}\left(p^{\prime}\right) N_{3 \lambda_{1} 3 \lambda_{2}}^{\lambda_{3}}(3 p) w^{\left(\lambda_{3}\right)} \cdot\left(\lambda_{3}^{\prime}-\kappa\left(\lambda_{1}+\lambda_{2}\right)\right),
$$

where $N_{\lambda_{1}^{\prime}, \lambda_{2}^{\prime}}^{\lambda_{3}^{\prime}}\left(p^{\prime}\right)$ is the integrable FR multiplicity at level $p^{\prime}-3$. 


\section{References}

[1] A. Belavin, A. Polyakov and A. Zamolodchikov, Nucl. Phys. B241 (1984) 333.

[2] E. Verlinde, Nucl. Phys. B300 [FS22] (1988) 360.

[3] H. Awata and Y. Yamada, Mod. Phys. Lett. A7 (1992) 1185.

[4] V.G. Kac, Infinite-dimensional Lie Algebras, third edition (Cambridge University Press, Cambridge 1990.

[5] M. Walton, Nucl.Phys. B 340 (1990) 777.

[6] P. Furlan, A.Ch. Ganchev and V.B. Petkova, Nucl. Phys. B 343 (1990) 205.

[7] B.L. Feigin and F.G. Malikov, Lett. Math. Phys. 31 (1994) 315; Modular functor and representation theory of sl(2) at a rational level, q-alg/9511011.

[8] V.G. Kac and M. Wakimoto, Proc. Natl. Sci. USA 85 (1988) 4956 ; V.G. Kac and M. Wakimoto, Adv. Ser. Math. Phys. 7 (1989) 138 ; V.G. Kac and M. Wakimoto, Acta Applicandae Math. 21 (1990) 3.

[9] V.G. Kac and D.A. Kazhdan, Adv. Math. 34 (1979) 97.

[10] F.G. Malikov, B.L. Feigin and D.B. Fuks, Funkt. Anal. Prilozhen. 20, no. 2 (1987) 25.

[11] P. Furlan, A.Ch. Ganchev and V.B. Petkova, Nucl. Phys. B 431 (1994) 622.

[12] V.K. Dobrev, Multiplet classification of the indecomposable highest weight modules over affine Lie algebras and invariant differential operators: the $A_{l}^{(1)}$ example, ICTP preprint IC/85/9.

[13] D.P. Zhelobenko, Compact Lie Groups and their Representations, (Amer. Math. Soc., Providence, 1973, translated from the Russian edition, Nauka, Moscow, 1970).

[14] V. Pasquier, J. Phys. A20 (1987) 5707.

[15] P. Di Francesco and J.-B. Zuber, Nucl. Phys. B338 (1990) 602;

P. Di Francesco and J.-B. Zuber, in Recent Developments in Conformal Field Theories, Trieste Conference 1989, S. Randjbar-Daemi, E. Sezgin and J.-B. Zuber eds., World Scientific 1990 ;

P. Di Francesco, Int. J. Mod. Phys. A7 (1992) 407.

[16] J.-B. Zuber, Comm. Math. Phys. 179 (1996) 265.

[17] E. Bannai, T. Ito, Algebraic Combinatorics I: Association Schemes, Benjamin/Cummings (1984).

[18] P. Furlan, A.Ch. Ganchev and V.B. Petkova, Nucl. Phys. B 491 [PM] (1997) 635. 